\title{
Two-dimensional weak pseudomanifolds on eight vertices
}

\author{
BASUDEB DATTA and NANDINI NILAKANTAN \\ Department of Mathematics, Indian Institute of Science, Bangalore 560 012, India \\ E-mail: dattab@math.iisc.ernet.in; nandini@ math.iisc.ernet.in
}

MS received 20 September 2001

\begin{abstract}
We explicitly determine all the two-dimensional weak pseudomanifolds on 8 vertices. We prove that there are (up to isomorphism) exactly 95 such weak pseudomanifolds, 44 of which are combinatorial 2-manifolds. These 95 weak pseudomanifolds triangulate 16 topological spaces. As a consequence, we prove that there are exactly three 8-vertex two-dimensional orientable pseudomanifolds which allow degree three maps to the 4-vertex 2-sphere.
\end{abstract}

Keywords. Two-dimensional complexes; pseudomanifolds; degree of a map.

\section{Introduction}

Recall that a simplicial complex (in short, complex) is a collection of non-empty finite sets such that every non-empty subset of an element is also an element. For $i \geq 0$, the elements of size $i+1$ are called the $i$-simplices of the complex. For $i=1,2$, the $i$-simplices are also called the edges and triangles of the complex, respectively. For a complex $X$, the maximum of $k$ such that $X$ has a $k$-simplex is called the dimension of $X$. The union of all the simplices of a complex $X$ is called the vertex-set of $X$ and is denoted by $V(X)$. Elements of $V(X)$ are called vertices of $X$. A complex $X$ is called finite if $V(X)$ is a finite set. A $k$-simplex $\left\{v_{0}, \ldots, v_{k}\right\}$ of a complex is also denoted by $v_{0} \cdots v_{k}$.

If $X_{1}$ and $X_{2}$ are two complexes, then a simplicial map from $X_{1}$ to $X_{2}$ is a map $\varphi$ : $V\left(X_{1}\right) \rightarrow V\left(X_{2}\right)$ such that $\sigma \in X_{1}$ implies $\varphi(\sigma) \in X_{2}$. A bijection $\pi: V\left(X_{1}\right) \rightarrow V\left(X_{2}\right)$ is called an isomorphism if both $\pi$ and $\pi^{-1}$ are simplicial. Two complexes $X_{1}, X_{2}$ are called (simplicially) isomorphic when such an isomorphism exists. We identify two complexes if they are isomorphic. An isomorphism from a complex $X$ to itself is called an automorphism of $X$. All the automorphisms of $X$ form a group, which is denoted by $\operatorname{Aut}(X)$. Two simplicial maps $f, g: X_{1} \rightarrow X_{2}$ are said to be equivalent (denoted by $f \cong g$ ) if there exist $\varphi \in \operatorname{Aut}\left(X_{1}\right)$ and $\psi \in \operatorname{Aut}\left(X_{2}\right)$ such that $\psi \circ f \circ \varphi=g$.

A $d$-dimensional simplicial complex $X$ is called a $d$-dimensional weak pseudomanifold if each simplex of $X$ is contained in a $d$-simplex of $X$ and each $(d-1)$-simplex of $X$ is contained in exactly two $d$-simplices of $X$. A $d$-dimensional weak pseudomanifold $X$ is called a pseudomanifold (without boundary) if for any pair $\sigma, \lambda$ of $d$-simplices of $X$, there exists a sequence $\tau_{1}, \ldots, \tau_{n}$ of $d$-simplices of $X$, such that $\sigma=\tau_{1}, \lambda=\tau_{n}$ and $\tau_{i} \cap \tau_{i+1}$ is a $(d-1)$-simplex of $X$ for $1 \leq i \leq n-1$. ( $P_{1}$ and $P_{2}$, given in $\S 2$, are pseudomanifolds but $Q_{1}$ and $Q_{2}$ are not.)

A simplicial complex is usually thought of as a prescription for the construction of a topological space by pasting together geometric simplices (see $\S 3$ for finite complexes, 
[8] and [11] for the general case). The space thus obtained from a complex $K$ is called the geometric carrier of $K$ and is denoted by $|K|$. We also say that $K$ triangulates $|K|$.

For any set $V$ on $d+2(\geq 2)$ elements, let $K$ be the simplicial complex whose simplices are all the non-empty proper subsets of $V$. Then $|K|$ is homeomorphic to the sphere $S^{d}$. This complex is called the standard $d$-sphere and is denoted by $S_{d+2}^{d}(V)$ or simply by $S_{d+2}^{d}$. A finite complex $X$ is called a combinatorial $d$-sphere, if $|X|$ is PL-homeomorphic to $\left|S_{d+2}^{d}\right|[10]$. Clearly, a finite one-dimensional complex is a combinatorial 1-sphere if and only if it is a pseudomanifold. For $n \geq 3$, the combinatorial 1 -sphere with $n$ vertices is unique and is denoted by $C_{n}$. The complex $C_{n}$ is also called an $n$-cycle. An $n$-cycle with edges $v_{1} v_{2}, \ldots, v_{n-1} v_{n}, v_{n} v_{1}$ is also denoted by $C_{n}\left(v_{1}, \ldots, v_{n}\right)$.

If $v$ is a vertex of a simplicial complex $X$, then the link of $v$ in $X$, denoted by $\operatorname{Lk}_{X}(v)$, is the complex whose simplices are those simplices $\tau$ of $X$ such that $v \notin \tau$ and $\{v\} \cup \tau$ is a simplex of $X$. The number of vertices in the link of $v$ is called the degree of $v$ and is denoted by $\operatorname{deg}(v)$. Clearly, the link of a vertex in a $d$-dimensional weak pseudomanifold is a $(d-1)$-dimensional weak pseudomanifold.

A finite simplicial complex $X$ is called a combinatorial $d$-manifold if $|X|$ is a $d$ dimensional PL-manifold (without boundary), i.e., $\operatorname{Lk}_{X}(v)$ is a combinatorial $(d-1)$ sphere for each vertex $v$ in $X[7,10]$. So, $X$ is a combinatorial 2-manifold if the link of each vertex is a cycle. We also know (e.g., see [7]) that a finite simplicial complex $K$ is a combinatorial 2-manifold if and only if $|K|$ is a two-dimensional topological manifold.

A vertex of a finite two-dimensional weak pseudomanifold is called singular if its link is not a cycle (and hence consists of more than one cycle). So, a two-dimensional weak pseudomanifold is not a combinatorial manifold if and only if it contains a singular vertex. (In each of $P_{1}, P_{2}, Q_{1}$ and $Q_{2}, 7$ is a singular vertex.)

A combinatorial 2-manifold $X$ is called $d$-equivelar if each vertex of $X$ has degree $d$. A combinatorial 2-manifold is called equivelar if it is $d$-equivelar for some $d$.

If the number of $i$-simplices of a $d$-dimensional finite complex $X$ is $f_{i}(X)(0 \leq i \leq d)$, then the number $\chi(X):=\sum_{i=0}^{d}(-1)^{i} f_{i}(X)$ is called the Euler characteristic of $X$.

If $K$ is a $d$-dimensional oriented pseudomanifold, then $\left[z_{K}\right]$ generates $H_{d}(K, \mathbb{Z})$, where $z_{K}:=\sum_{\sigma^{d} \in K} 1 \cdot \sigma^{d}$ (summation is taken over all the positively oriented $d$-simplices). Let $K$ and $L$ be two oriented $d$-dimensional pseudomanifolds. If $\varphi: K \rightarrow L$ is a simplicial map then $\varphi_{d}^{*}: H_{d}(K, \mathbb{Z}) \rightarrow H_{d}(L, \mathbb{Z})$ is a homomorphism and hence there exists $m \in \mathbb{Z}$ such that $\varphi_{d}^{*}\left(\left[z_{K}\right]\right)=m\left[z_{L}\right]$. This $m$ is called the degree of $\varphi$ and is denoted by $\operatorname{deg}(\varphi)$ [11].

Let $K$ be a two-dimensional pseudomanifold and $\varphi: K \rightarrow S_{4}^{2}$ be a simplicial map. If $\varphi(u) \neq \varphi(v)$ for each edge $u v$ of $K$ then $\varphi$ is called a 4-coloring [12].

It is known (e.g., see [3,5]) that if the number of vertices of a two-dimensional weak pseudomanifold $M$ is at most 6 then $M$ is a combinatorial 2-manifold and $M$ is isomorphic to $S_{1}, \ldots, S_{4}$ or $R_{1}$ (given in $\S 2$ ).

In [5], we have seen that there are exactly nine 7-vertex combinatorial 2-manifolds and four 7-vertex two-dimensional weak pseudomanifolds which are not combinatorial 2-manifolds. Among the four non-manifolds two are pseudomanifolds, which triangulate the pinched sphere (the space obtained by identifying two points of $S^{2}$ ).

In [6], we have determined all the equivelar combinatorial 2-manifolds on at most 11 vertices. There are 27 such equivelar combinatorial 2-manifolds.

Altshuler and Steinberg [2] showed that there are fourteen 8-vertex combinatorial 2spheres. Cervone [4] showed that there are exactly six 8-vertex combinatorial 2-manifolds, which triangulate the Klein bottle. It is known (e.g., see [7,9]) that there does not exist 
any 8-vertex combinatorial 2-manifold of Euler characteristic -1 . Here, we classify all the two-dimensional weak pseudomanifolds on 8 vertices. More explicitly, we prove:

Theorem 1.1. There are exactly 44 distinct combinatorial 2-manifolds on 8 vertices, namely, $S_{10}, \ldots, S_{23}, R_{5}, \ldots, R_{20}, T_{2}, \ldots, T_{8}, K_{1}, \ldots, K_{6}$ and $D$ (given in $\S 2$ ).

Theorem 1.2. There are exactly 51 distinct 8-vertex two-dimensional weak pseudomanifolds which are not combinatorial 2-manifolds, namely, $P_{3}, \ldots, P_{39}, Q_{3}, \ldots, Q_{16}$ (given in $\S 2$ ).

\section{COROLLARY 1.3}

Let $M$ be a two-dimensional weak pseudomanifold on $n(\leq 8)$ vertices.

(i) If $|M|$ is a manifold then $|M|$ is homeomorphic to the 2-sphere $\left(S^{2}\right)$, the real projective plane $\left(\mathbb{R} P^{2}\right)$, the torus $\left(S^{1} \times S^{1}\right)$, the Klein bottle $(K)$ or the space consisting of two disjoint 2-spheres.

(ii) If $|M|$ is not a manifold then $|M|$ is homeomorphic to the pinched sphere $(P), \mathbb{R} P^{2} \# P$, $P \# P, \mathbb{R} P^{2} \# P \# P, K \# P,\left(S^{1} \times S^{1}\right) \# P$, the union of two $S^{2}$ 's having one, two, three or four points in common or the union of $S^{2}$ and $\mathbb{R} P^{2}$ having three points in common (given at the end of §2). (Here, $A \# B$ denotes the connected sum of $A$ and $B$ ).

If $\varphi: K \rightarrow S_{4}^{2}$ is a simplicial map, where $K$ is an oriented 8-vertex two-dimensional pseudomanifold, then $f_{2}(K) \leq 18$ and hence $\operatorname{deg}(\varphi) \leq 4$. Here we prove:

Theorem 1.4. Let $\varphi: K_{n}^{2} \rightarrow S_{4}^{2}$ be a simplicial map, where $K_{n}^{2}$ is a two-dimensional oriented pseudomanifold on $n$ vertices. Let $f, g$ and $h$ be as in Example 2.1. If $n \leq 8$ then $\operatorname{deg}(\varphi) \leq 3$ (and hence $\geq-3$ ). Equality is attained here if and only if $\varphi$ is equivalent to $f, g$ or $h$.

Remark 1.5. Observe that $P_{3}, \ldots, P_{39}$ (in Theorem 1.2) are pseudomanifolds, whereas $Q_{3}, \ldots, Q_{16}$ are not pseudomanifolds. Among the pseudomanifolds, $P_{3}, \ldots, P_{20}$, $P_{28}, \ldots, P_{36}$ and $P_{39}$ are orientable and $P_{21}, \ldots, P_{27}, P_{37}$ and $P_{38}$ are non-orientable.

Remark 1.6. If $M$ is an 8-vertex two-dimensional weak pseudomanifold, then it is easy to see that $\chi(M)$ lies between -1 and 4 . If $M$ is a combinatorial 2-manifold then, from Theorem 1.1, $\chi(M)$ is $0,1,2$ or 4 . However, by Theorem 1.2, there exist weak pseudomanifolds with Euler characteristic $-1, \ldots, 3$.

Remark 1.7. Theorem 1.1, Theorem 1.2 and Proposition 3.1 together with the results in [3], which determine all the $d$-dimensional weak pseudomanifolds on at most $d+4$ vertices, classify all the $d$-dimensional $(d \neq 3)$ weak pseudomanifolds on less than or equal to 8 vertices. Moreover, Theorem 1.1 together with Proposition 3.1, the results in [1], which classify all the combinatorial 3-manifolds on at most 8 vertices and the results in [3] classify all the combinatorial manifolds on less than or equal to 8 vertices.

In $\S 2$ we present all the two-dimensional weak pseudomanifolds on at most 8 vertices. In $\S 3$ we give some definitions, constructions and results which we shall need later. In $\S 4$ we consider combinatorial manifolds and prove Theorem 1.1. In $\S 5$ we consider weak pseudomanifolds which are not combinatorial manifolds and prove Theorem 1.2. In $\S 6$ we prove Corollary 1.3 and Theorem 1.4. 


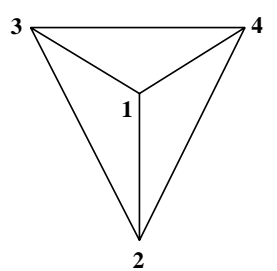

$S_{1}\left(3^{4}\right)$

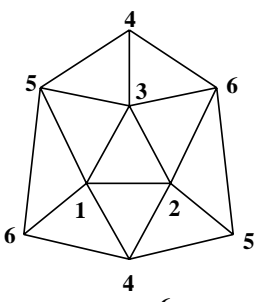

$R_{1}\left(5^{6}\right)$

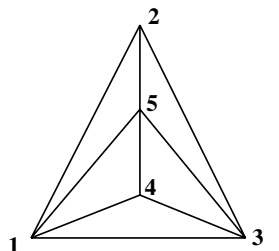

$S_{2}\left(4.3^{2}\right)$

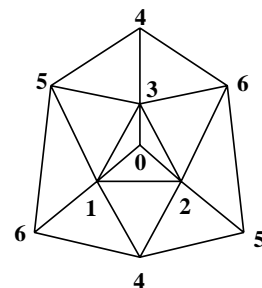

$R_{2}(6.5 .3)$

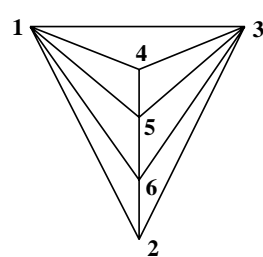

$\mathrm{S}_{3}\left(\mathbf{5 . 4 . 3}^{2}\right)$

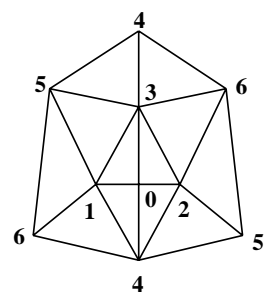

$R_{3}(6.5 .4)$

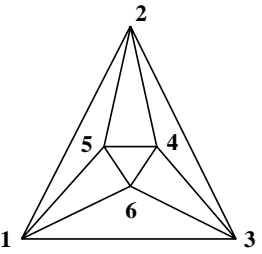

$S_{4}\left(4^{6}\right)$

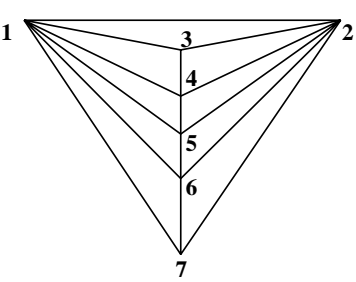

$S_{5}\left(6^{2.4 .3} .^{2}\right)$

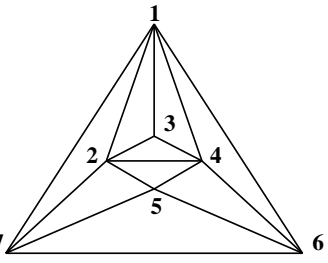

$\mathrm{S}_{8}$

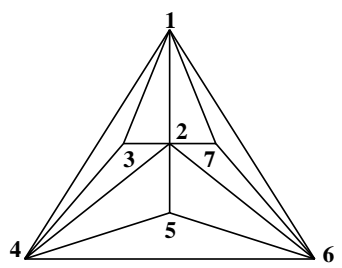

$S_{6}\left(6.5^{3} 3^{3}\right)$

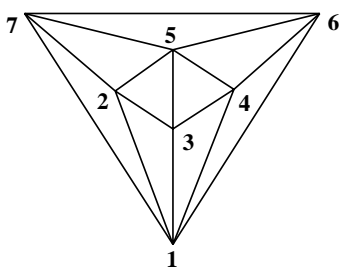

$S_{9}\left(5^{2} 4^{5}\right)$
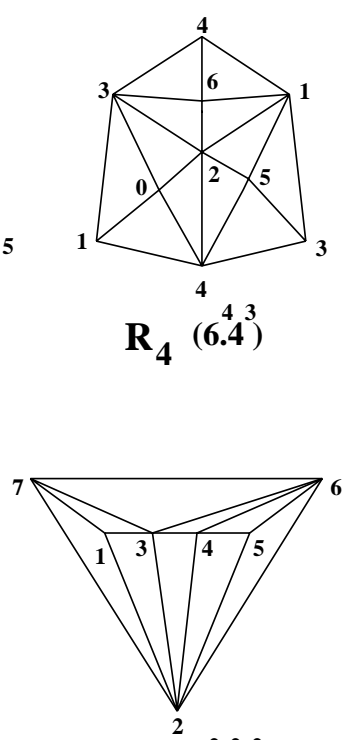

$\mathrm{S}_{7}\left(\mathbf{6 . 5 . 4 . 3 ^ { 2 } )}\right.$

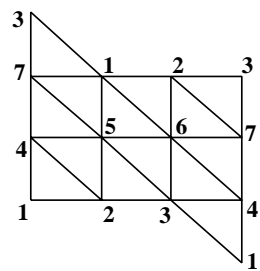

$\mathrm{T}_{1}\left(6^{7}\right)$

\section{Examples}

In Theorems 1.1 and 1.2 we have stated that there are 95 two-dimensional weak pseudomanifolds on 8 vertices. In this section we present all these 95 weak pseudomanifolds. We also present all the 18 two-dimensional weak pseudomanifolds on less than or equal to 7 vertices. The degree sequences are presented parenthetically below the figures. For $0 \leq i \leq 7, i$ in the figures represents the vertex $v_{i}$. At the end of this section, we present 


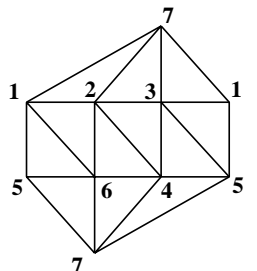

$P_{1}\left(6.5^{6}\right)$

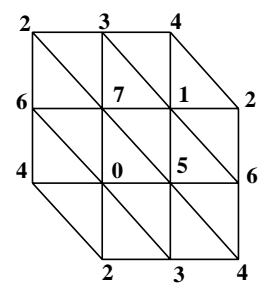

$\mathbf{T}_{2}\left(\mathbf{6}^{8}\right)$

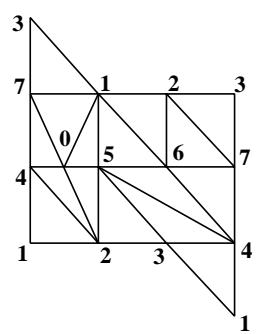

$T_{6}\left(7^{3} .6^{2} .5^{3}\right)$

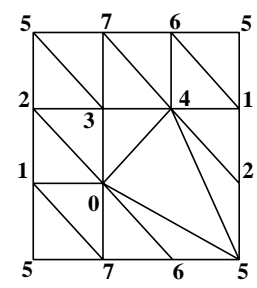

$K_{2}\left(\mathbf{7 . 6 . 5}^{3}\right)$

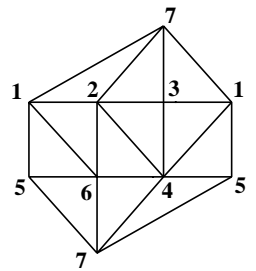

$P_{2}\left(6^{3.5 .2}\right)$

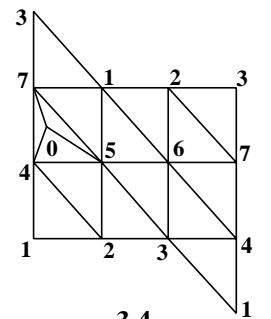

$T_{3}(\mathbf{7 . 6 . 3})$

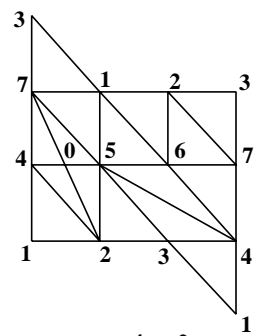

$\mathbf{T}_{7}(\mathbf{7 . 6 . 5 . 4 )}$

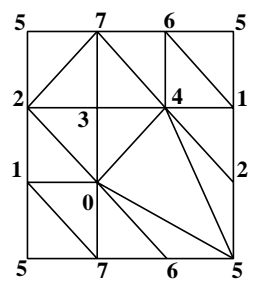

$K_{3}{ }^{(7.6 .5 .4)}$

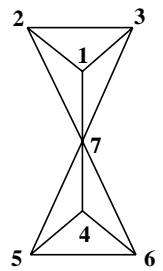

$Q_{1}{ }^{\left(6.3^{6}\right)}$

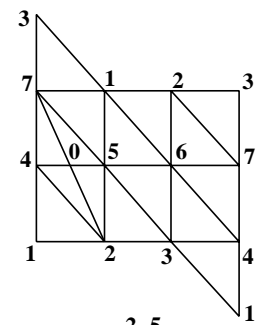

$T_{4} \quad\left(\mathbf{7 . 6 . 4}^{2}\right)$

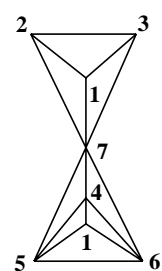

$\left.Q_{2}{ }^{\left(6.4 .3^{2}\right.}\right)$

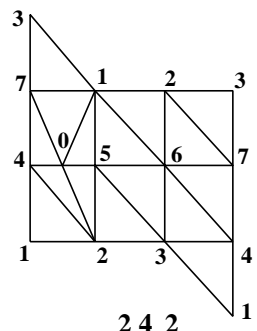

$T_{5}\left(7^{2.6 .5}\right)$
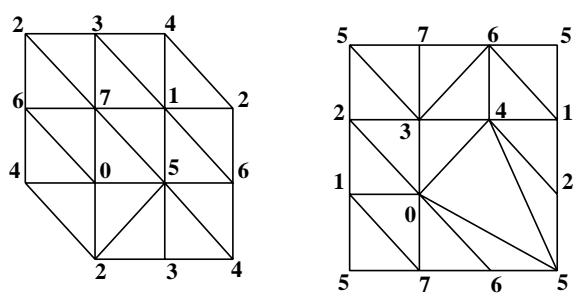

$T_{8}\left(7^{2} .^{4} 5^{2}\right)$

$K_{1}\left(7^{2} .6^{4} .5^{2}\right)$
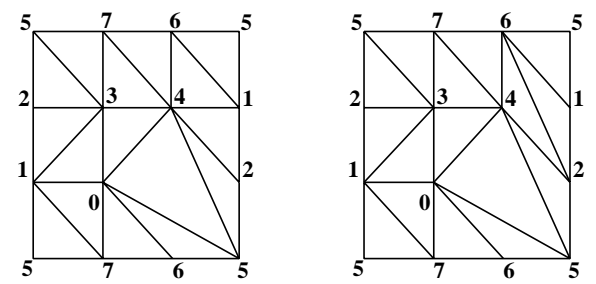

$\mathrm{K}_{4} \quad\left(\mathbf{7 . 6 . 5}^{3}\right)$

$K_{5} \quad(\mathbf{7 . 6 . 5})$

the geometric carriers of all the weak pseudomanifolds on 8 vertices. At the beginning we present three degree 3 maps (which we have mentioned in Theorem 1.4) to the 4-vertex 2-sphere.

Example 2.1. Let $S_{1}=S_{4}^{2}(\{a, b, c, d\})$ with the positively oriented 2-simplices $a b c, a c d$, $a d b, b d c$. Let $S_{15}, P_{34}$ and $P_{35}$ be as given below: 


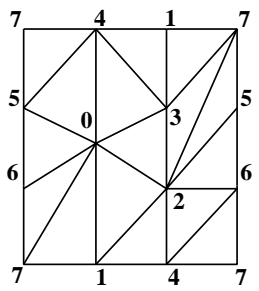

$K_{6}\left(7^{4.5}\right)$

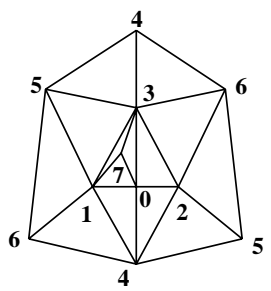

$R_{8}{ }^{(7.6 .5 .3)}$

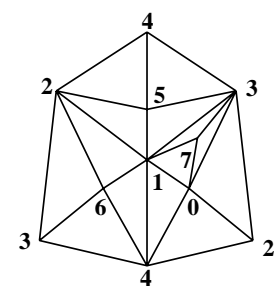

$R_{12}{ }^{\left(^{2} \cdot{ }^{2} \cdot 6.5 .4 .3\right)}$

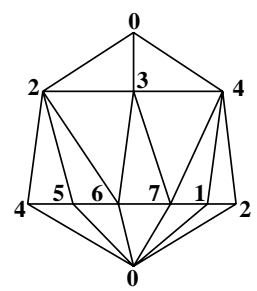

$\left.R_{16}{ }^{\left(7.6 .5 .4^{2}\right.}\right)$

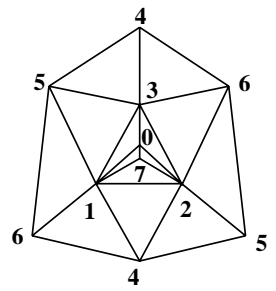

$\mathbf{R}_{5}{ }^{(7.6 .5 .4 .3)}$

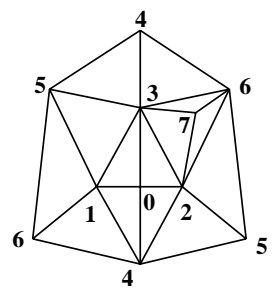

$R_{9}{ }^{(7.6 .5 .4 .3)}$

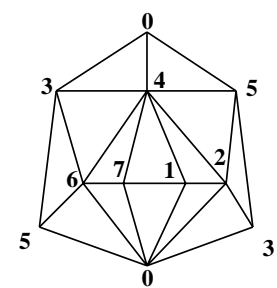

$$
R_{13}{ }^{\left(7.5 .4^{2}\right)}
$$

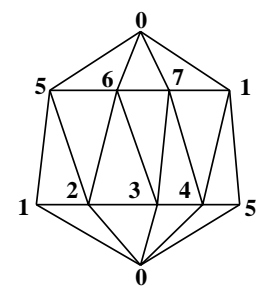

$\mathbf{R}_{17}\left(7.5^{7}\right)$

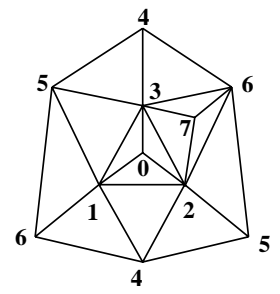

$\mathbf{R}_{6}\left(7^{2} \cdot .^{2} \cdot .^{2} \cdot 3^{2}\right)$

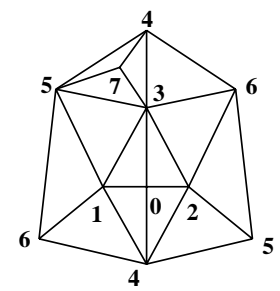

$R_{10}{ }^{(7.6 .5 .4 .3)}$
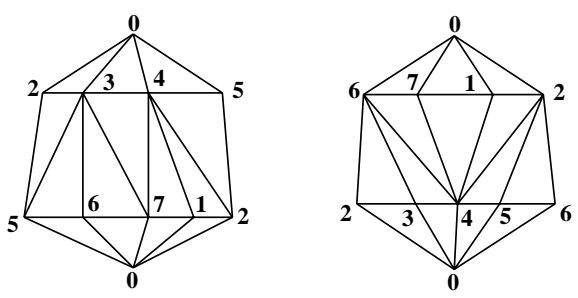

$\left.R_{14}{ }^{\left(7.6 .5 .4^{2}\right.}\right)$

$$
\left.R_{15}{ }^{\left(7^{2} .6 .4^{4}\right.}\right)
$$

(a) Consider the orientation on $S_{15}$ given by the positively oriented 2-simplices 176, 160, $064,104,143,132,234,524,546,562,267,127$. Let $f: S_{15} \rightarrow S_{1}$ be the simplicial map given by $f(1)=f(5)=a, f(0)=f(2)=b, f(4)=f(7)=c$ and $f(3)=$ $f(6)=d$. 


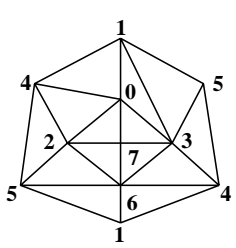

$\mathbf{R}_{20}{ }^{(6.5 .4)}$

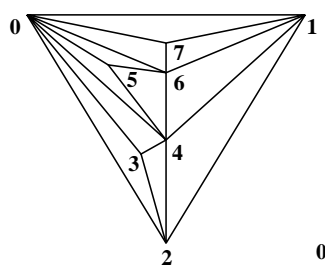

$S_{10}\left(\mathbf{7 . 6 . 5 . 4 . 3 ^ { 3 }}\right)$

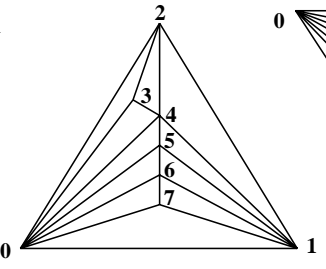

$S_{11}\left(7.6 .5 .4 .3^{2}\right)$

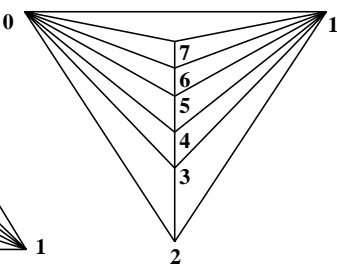

$S_{12}{\left(7.4 .3^{2}\right)}^{2}$
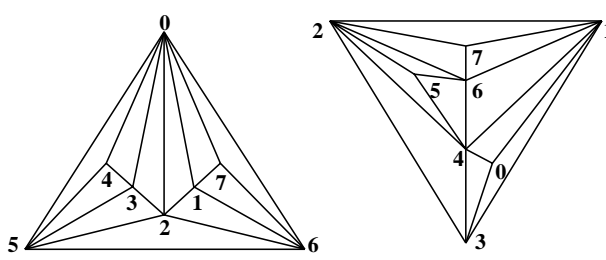

$$
\left.S_{13}{ }^{\left(7.5 .4 .3^{3}\right)} \quad S_{14}{ }^{\left(6.5 .4 .3^{3}\right.}\right)
$$

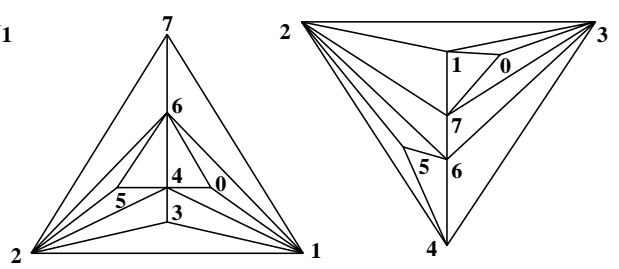

$$
\left.S_{15}{ }^{\left(6^{4} 3^{4}\right)} \quad S_{16}{ }^{\left(6^{2} .5^{2} .3^{2}\right.}\right)
$$

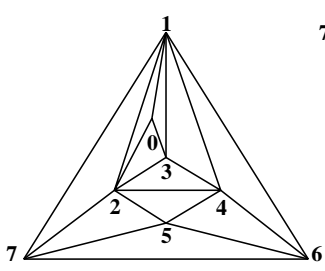

$S_{17}\left(6^{2} .5 .4 .3\right)$

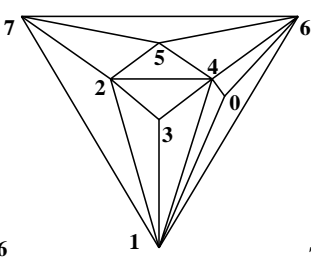

$S_{18}{ }^{\left(6^{2} \cdot 5^{2} \cdot 4^{2} \cdot 3^{2}\right)}$

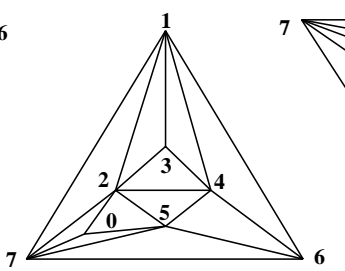

$S_{19}\left(6.5 .4 .3^{2}\right)$

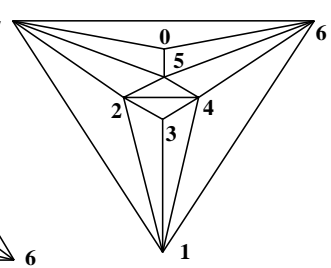

$S_{20}\left(5^{6.2}\right)$

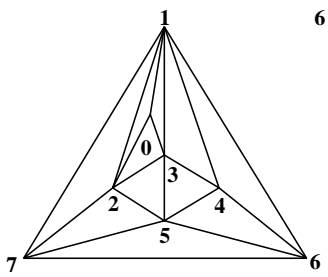

$S_{21}(6.5 .4 .3)$

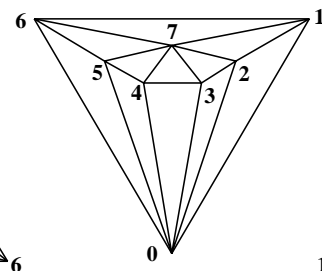

$S_{22}\left(6^{2.4}\right)$

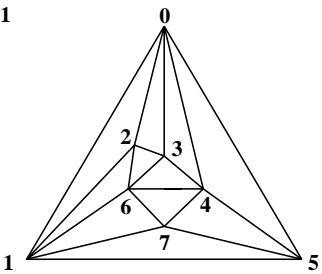

$S_{23}^{\left(5.4^{4}\right)}$

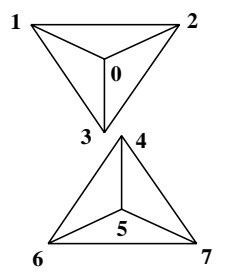

D $\left(3^{8}\right)$

(b) Consider the orientation on $P_{34}$ given by the positively oriented 2-simplices 475,467 , 765, 056, 015, 061, 162, 426, 024, 043, 453, 135, 173, 037, 072, 127. Let $g: P_{34} \rightarrow S_{1}$ be the simplicial map given by $g(0)=g(4)=a, g(1)=g(7)=b, g(2)=g(5)=c$ and $g(3)=g(6)=d$.

(c) Consider the orientation on $P_{35}$ given by the positively oriented 2-simplices 523, 537, 572, 274, 704, 760, 673, 163, 130, 203, 102, 124, 146, 564, 506, 540. Let $h: P_{35} \rightarrow S_{1}$ be the simplicial map given by $h(1)=h(5)=a, h(2)=h(6)=b, h(3)=h(4)=c$ and $h(0)=h(7)=d$. 


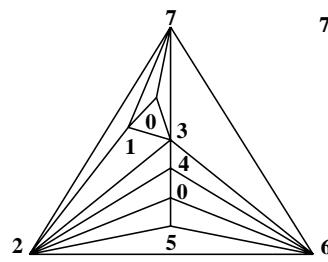

$P_{3} \quad\left(7^{2} .6 .5 .4 .3\right)$

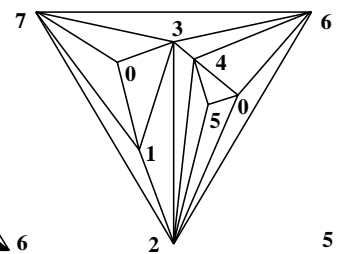

$\mathbf{P}_{4}$ (7.6.5.4.3)

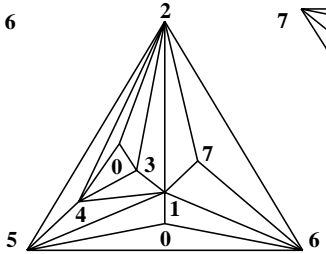

$P_{5}\left(\mathbf{7 . 6 . 5 . 4 . 3 )}^{2}\right.$

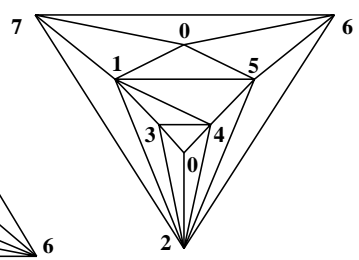

$P_{6} \quad\left(7.6 .5^{2} .^{3}\right)$

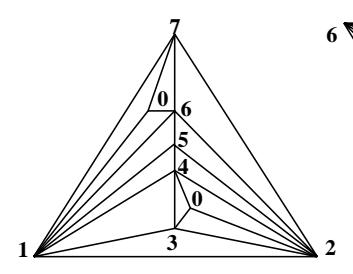

$P_{7} \quad\left(7.6 .5 .4^{2}\right)$

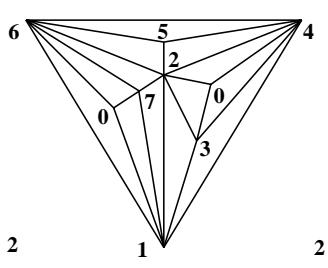

$P_{8} \quad(7.6 .4 .3)$

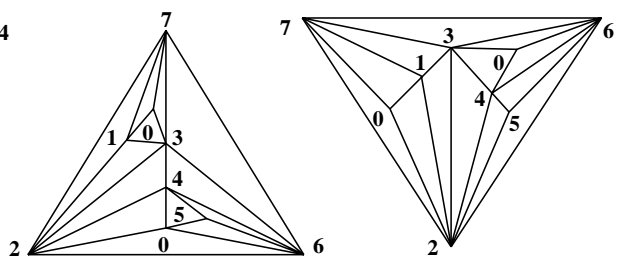

$P_{9} \quad(7.6 .5 .4 .3)$

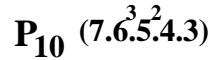
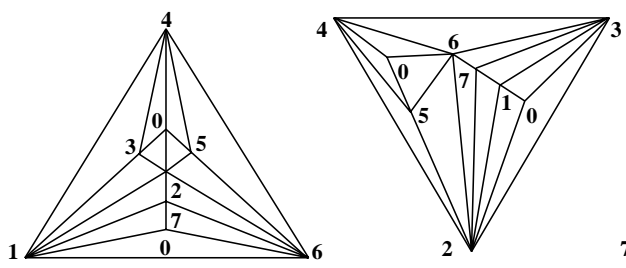

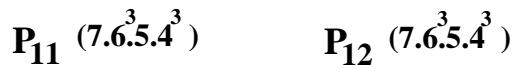

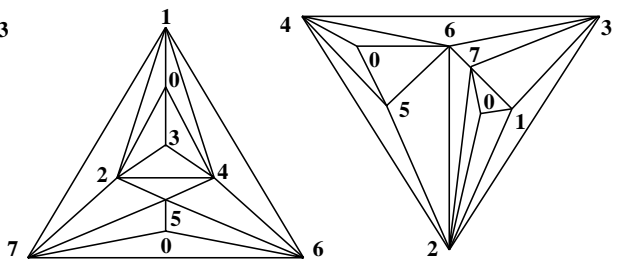

$$
\left.P_{13}{ }^{(7.6 .5 .3)} \quad P_{14}{ }^{(7.6 .5 .4}\right)
$$

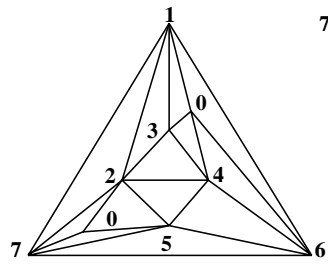

$P_{15}$ (7.6.5.4)

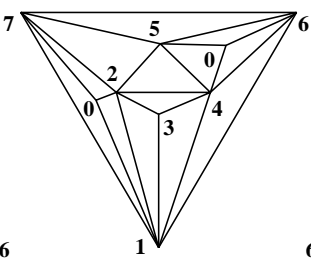

$P_{16}\left(6^{4.5 .3}\right)$

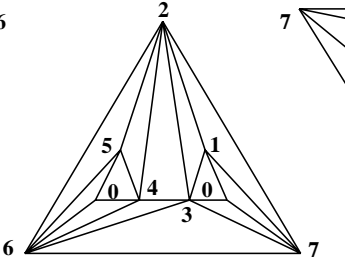

$P_{17}\left(6^{4} .5^{2} .^{2}\right)$

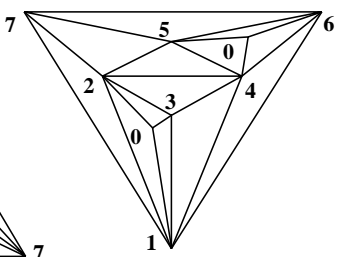

$P_{18}\left(6^{4} .5^{2} 4^{2}\right)$

Then $\operatorname{deg}(f)=\operatorname{deg}(g)=\operatorname{deg}(h)=3$. The map $f$ is a 4-coloring but $g$ and $h$ are not.

\section{Preliminaries}

For a finite simplicial complex $X$, if $n_{i}(>0)$ is the number of vertices of degree $d_{i}$ and $d_{1}>d_{2}>\cdots$, then $d_{1}^{n_{1}} \ldots \ldots d_{k}^{n_{k}}$ is called the degree sequence of $X$, where $\sum_{i=1}^{k} n_{i}$ is equal to the number of vertices of $X$. 


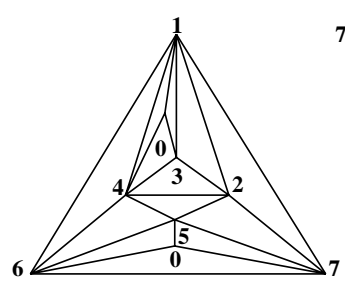

$P_{19}(6.5 .4)$

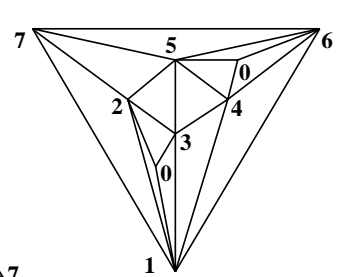

$\mathbf{P}_{20}(6.5 .4)$

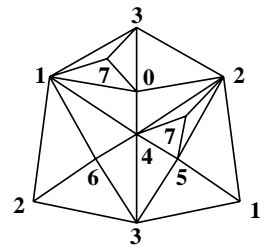

$P_{21}\left(7^{4} 6.5 .4\right)$

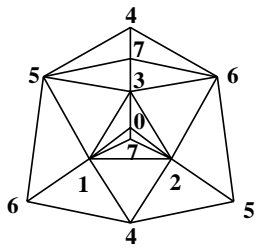

$P_{22}($ 7.6.5.4)

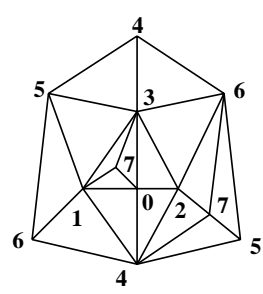

$P_{23}\left(7^{3} .6 .5\right)$

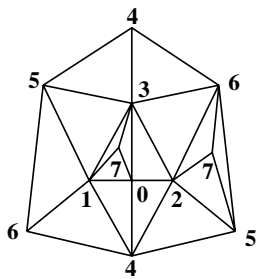

$P_{27}$ (7.6.5)

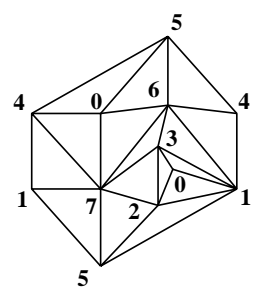

$\mathbf{P}_{31}\left(\mathbf{7 . 6 . 5}^{3}\right)$

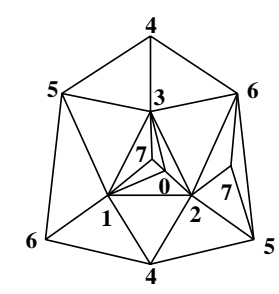

$\mathbf{P}_{24}{ }^{(7.6 .5 .4)}$

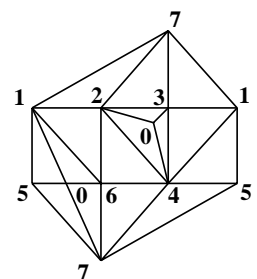

$\mathbf{P}_{28}{ }^{(7.6 .5 .4)}$

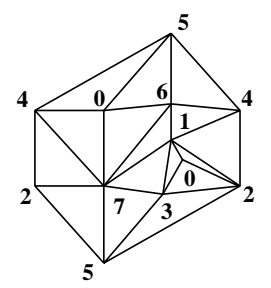

$\left.\mathbf{P}_{32}{ }^{\left(7.6 .5^{2}\right.}\right)$

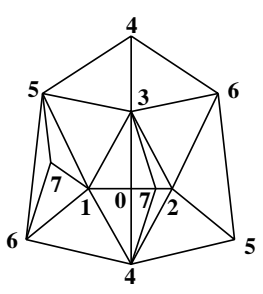

$P_{25}{ }^{(7.6 .5 .4)}$

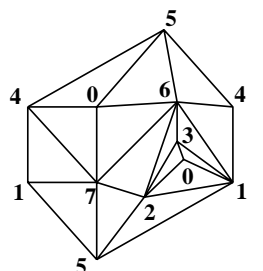

$P_{29}(\mathbf{7 . 6 . 5 . 4})$

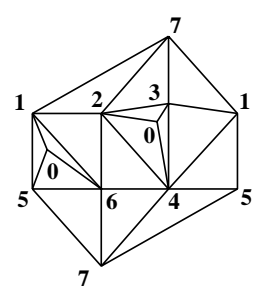

$\mathbf{P}_{33}\left(7^{2} .6 .5^{4}\right)$

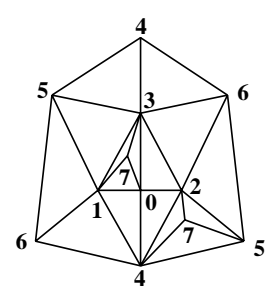

$P_{26}\left(\mathbf{7 . 6 . 5}^{2.2}\right)$

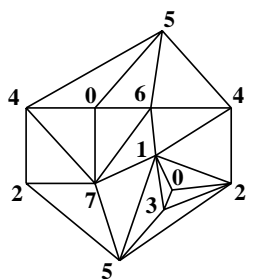

$\mathbf{P}_{30}$ (7.6.5.4)

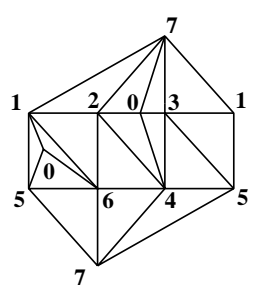

$\mathbf{P}_{34}\left(\mathbf{7 . 6 . 5}^{2}\right)$

If $m_{i}(>0)$ is the number of singular vertices of degree $c_{i}$ in a two-dimensional weak pseudomanifold $X$ and $c_{1}>c_{2}>\cdots$ then $c_{1}^{m_{1}} \ldots \ldots c_{k}^{m_{k}}$ is called the singular degree sequence of $X$, where $\sum_{i=1}^{k} m_{i}$ is equal to the number of singular vertices of $X$.

If $X$ is a finite simplicial complex then one defines a geometric realization of $X$ as follows: Let $V(X)=\left\{v_{1}, \ldots, v_{n}\right\}$. We choose a set of $n$ points $\left\{x_{1}, \ldots, x_{n}\right\}$ in $\mathbb{R}^{N}$ (for some $N$ ) in such a way that a subset $S=\left\{x_{j_{1}} \ldots, x_{j_{i+1}}\right\}$ of $i+1$ points is affinely independent if $\sigma=v_{j_{1}} \cdots v_{j_{i+1}}$ is a simplex of $X$. The convex set spanned by $S$ is called the geometric carrier of $\sigma$ or the geometric simplex corresponding to $\sigma$ and denoted 


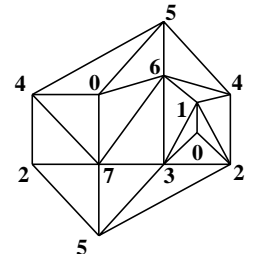

$\mathbf{P}_{35}{ }^{(7.6 .5)}$

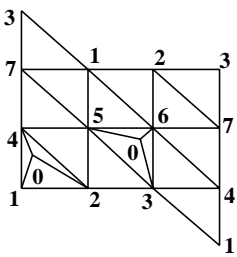

$P_{39}\left(7^{6} \cdot 6^{2}\right)$

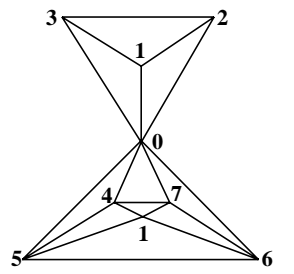

$Q_{6}{ }^{\left(7^{2} .4 .3^{2}\right)}$

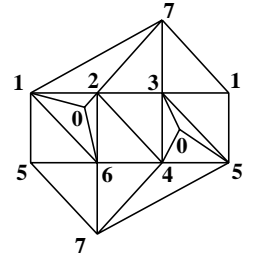

$\mathbf{P}_{36}\left(\mathbf{6}^{8}\right)$

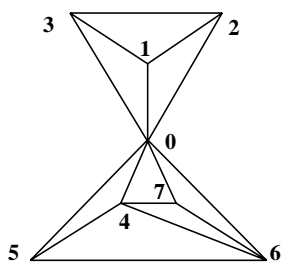

$Q_{3}\left(7.4^{2} 3^{5}\right)$

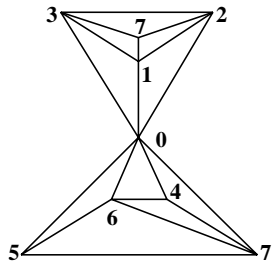

$\left.Q_{7}{ }^{(7.4 .3} \mathbf{3}^{2}\right)$

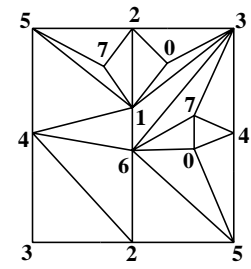

$\mathbf{P}_{37}\left(7^{6.6} 6^{2}\right)$

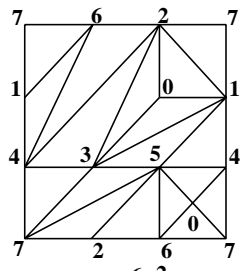

$\left.\mathbf{P}_{38}{ }^{\left(7^{6} 6^{2}\right.}\right)$

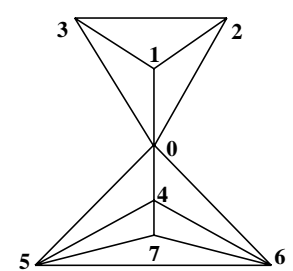

$Q_{4}{ }^{\left(6.4^{3} 3^{4}\right)}$

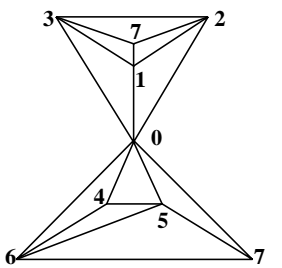

$Q_{8}{ }^{\text {(7.6.4.3) }}$

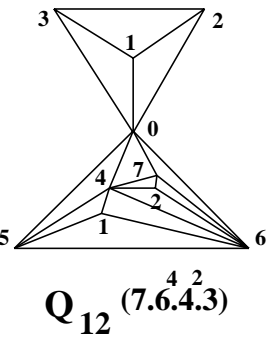

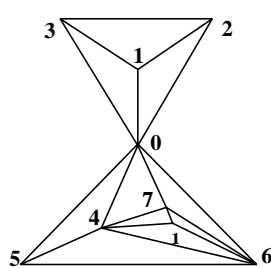

$Q_{5}\left(7.6 .5 .4 .3^{3}\right)$

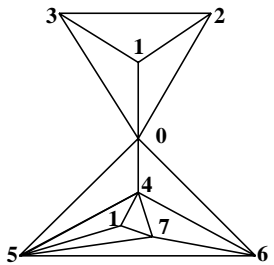

$Q_{9}\left(6^{2} .5^{2} \cdot 3^{2} 3^{2}\right)$
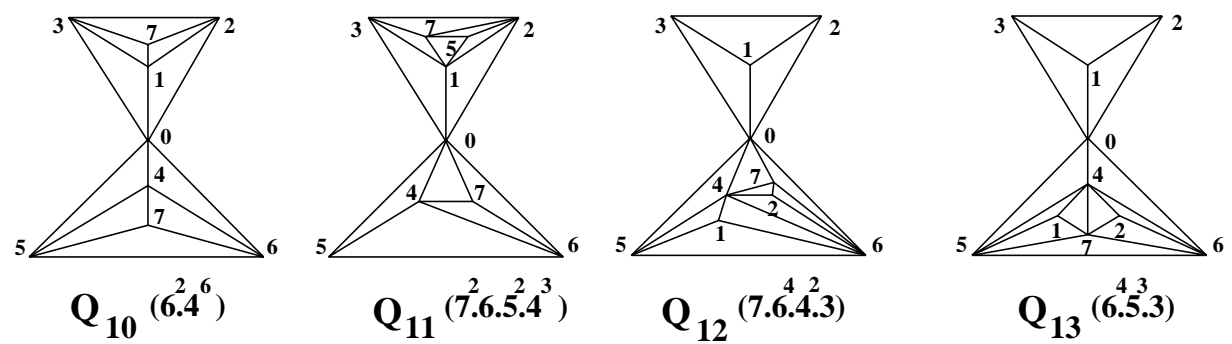

by $|\sigma|$. Since $X$ is finite we can choose $N$ so that $\sigma \cap \gamma=\emptyset$ implies $|\sigma| \cap|\gamma|=\emptyset$. The set $\mathcal{X}:=\{|\sigma|: \sigma \in X, \sigma \cap \gamma=\emptyset \Rightarrow|\sigma| \cap|\gamma|=\emptyset\}$ is called a geometric simplicial complex corresponding to $X$ or a geometric realization of $X$. The topological space $|X|:=\cup_{\sigma \in X}|\sigma|$ is called a geometric carrier of $X$. Clearly, if two finite complexes have a common geometric realization, then they are isomorphic and isomorphic finite complexes have homeomorphic geometric carriers [8]. We identify a complex with its geometric realization. 


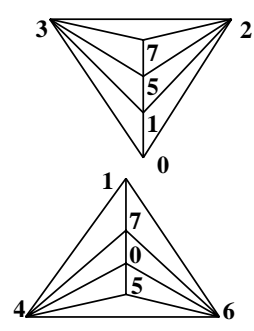

$Q_{14}^{\left(7.5^{4}\right)}$

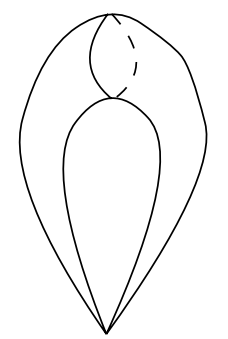

$\mathbf{P}$

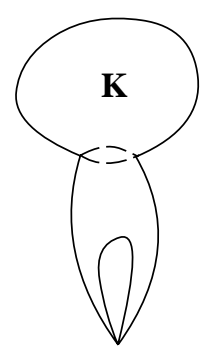

$\mathbf{K} \# \mathbf{P}$
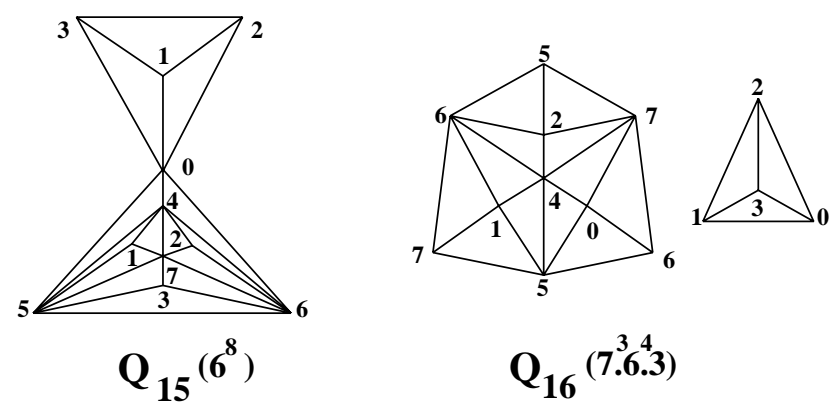

$$
Q_{16}^{(7.6 .3)}
$$
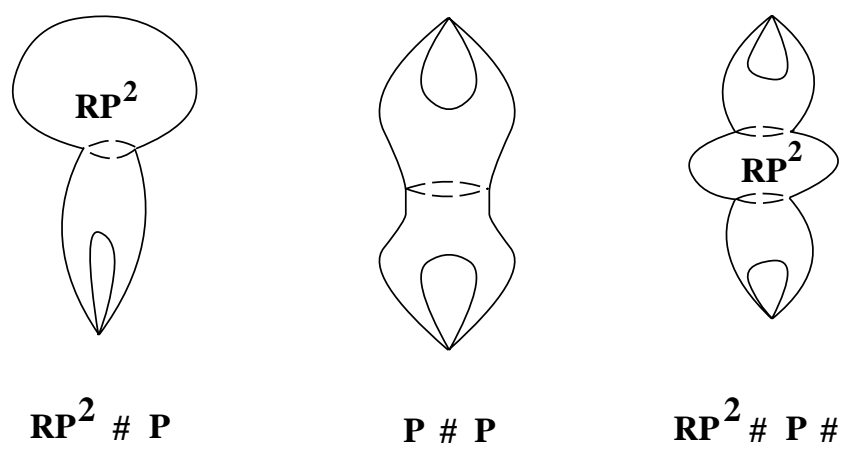

$\mathbf{P}$ \# $\mathbf{P}$
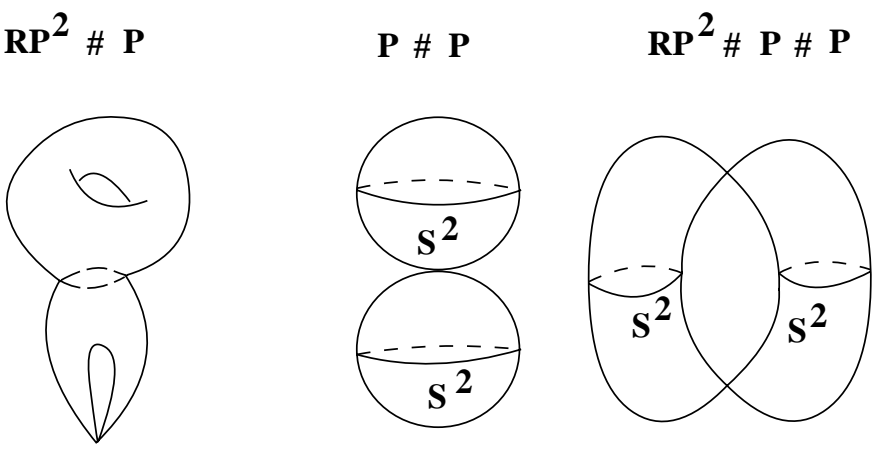

$(\mathbf{S} \times \mathbf{S}) \# \mathbf{P}$
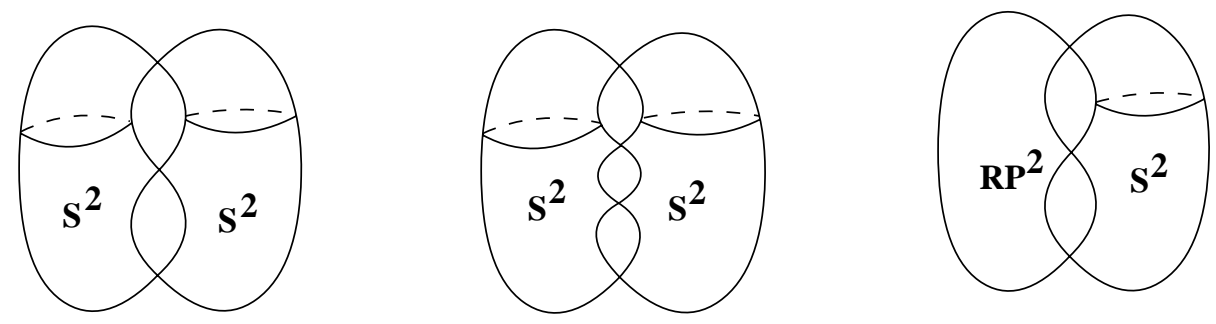

If $M$ is an $n$-vertex two-dimensional weak pseudomanifold with $f_{1}$ edges and $f_{2}$ triangles then $3 f_{2}=2 f_{1}$. Therefore,

$$
\chi(M)=n-f_{1}+f_{2}=n-\frac{f_{1}}{3}=n-\frac{f_{2}}{2} .
$$


In [5], we have seen the following:

\section{PROPOSITION 3.1}

There are exactly 13 distinct two-dimensional weak pseudomanifolds on 7 vertices, namely, $T_{1}, R_{2}, R_{3}, R_{4}, S_{5}, \ldots, S_{9}, P_{1}, P_{2}, Q_{1}$ and $Q_{2}$.

Altshuler and Steinberg [2] showed the following:

\section{PROPOSITION 3.2}

There are exactly 14 distinct combinatorial 2-spheres on 8 vertices, namely, $S_{10}, \ldots, S_{23}$.

If $X_{1}, X_{2}$ are two simplicial complexes with disjoint vertex sets, then their join $X_{1} * X_{2}$ is the complex whose simplices are those of $X_{1}$ and $X_{2}$ and the unions of simplices of $X_{1}$ with simplices of $X_{2}$. If both $X_{1}$ and $X_{2}$ are pseudomanifolds then so is $X_{1} * X_{2}$ [3]. Observe that $S_{2}^{0}(\{a, b\}) * S_{2}^{0}(\{c, d\})=C_{4}(a, c, b, d)$.

If $M$ is a two-dimensional simplicial complex and $\tau=a b c$ is a 2-simplex then $(M \backslash \tau) \cup$ $(\partial \tau * v)$ denotes the two-dimensional complex whose 2-simplices are $a b v, a c v, b c v$ and those of $M$ other than $\tau$, where $v \notin V(M)$. This complex is said to be obtained from $M$ by starring the vertex $v$ in $\tau$. Observe that $R_{2}$ is obtained from $R_{1}$ by starring the vertex 0 in 123. Similarly, if $a b$ is an edge (contained in $a b c$ and $a b d$ ) and $v \notin V(M)$ then $(M ! \backslash\{a b\}) \cup\left(C_{4}(a, c, b, d) * v\right)$ denotes the two-dimensional complex whose 2-simplices are $a c v, a d v, b c v, b d v$ and those of $M$ other than $a b c$ and $a b d$. This complex is said to be obtained from $M$ by starring the vertex $v$ in the edge $a b$. The complex $R_{3}$ is obtained from $R_{1}$ by starring the vertex 0 in the edge 12 .

Let $M, N$ be two simplicial complexes with $\sigma_{1}, \ldots, \sigma_{m} \in M$ and $\tau_{1}, \ldots, \tau_{m} \in N$. We say $\left(M, \sigma_{1}, \ldots, \sigma_{m}\right)$ and $\left(N, \tau_{1}, \ldots, \tau_{m}\right)$ are isomorphic (denoted by $\left(M, \sigma_{1}, \ldots, \sigma_{m}\right)$ $\left.\cong\left(N, \tau_{1}, \ldots, \tau_{m}\right)\right)$ if there exists an isomorphism $\varphi$ from $M$ to $N$ such that $\varphi\left(\sigma_{i}\right)=\tau_{i}$ for $1 \leq i \leq m$.

Let $M$ be a $d$-dimensional weak pseudomanifold. Let $u$ and $v$ be two distinct vertices of $M$ such that $u v$ is not an edge. If $V(\operatorname{Lk}(u)) \cap V(\operatorname{Lk}(v))=\emptyset$ then define the complex $\widetilde{M}=\{\tau \in M: u \notin \tau, v \notin \tau\} \cup\{(\tau \backslash\{u\}) \cup\{w\}: u \in \tau\} \cup\{(\tau \backslash\{v\}) \cup\{w\}: v \in \tau\}$. This $\widetilde{M}$ is called the simplicial complex obtained from $M$ by identifying $u$ and $v$ (to a new vertex $w$ ). Observe that $P_{1}$ is obtained from $S_{20}$ by identifying vertices 0 and 3 of $S_{20}$ and $P_{2}$ is is obtained from $S_{16}$ by identifying vertices 0 and 5 of $S_{16}$.

If $\tau_{1}=a b c$ and $\tau_{2}=x y z$ are two disjoint 2-simplices of a two-dimensional simplicial complex $M$ and $v \notin V(M)$ then $\left(M \backslash\left\{\tau_{1}, \tau_{2}\right\}\right) \cup\left(\left(\partial \tau_{1} \cup \partial \tau_{2}\right) * v\right)$ denotes the complex whose 2-simplices are $a b v, a c v, b c v, x y v, y z v, x z v$ and those of $M$ other than $\tau_{1}$ and $\tau_{2}$. Observe that we get $P_{33}$ from $P_{2}$ and $P_{39}$ from $T_{1}$ by this process.

From these definitions one gets the following:

\section{PROPOSITION 3.3}

Let $\tilde{M}$ be obtained from $M$ by identifying two vertices $u$ and $v$.

(a) If $M$ is a (weak) pseudomanifold, then so is $\tilde{M}$.

(b) If $\widetilde{N}$ is obtained from $N$ by identifying two vertices $u_{1}$ and $v_{1}$ and $\left(N,\left\{u_{1}\right\},\left\{v_{1}\right\}\right)$ $\cong(M,\{u\},\{v\})$, then $\widetilde{N} \cong \widetilde{M}$.

(c) If $M$ is a two-dimensional pseudomanifold and both $u$ and $v$ are non-singular then $|\widetilde{M}|$ is homeomorphic to the connected sum of $|M|$ and the pinched sphere. 


\section{PROPOSITION 3.4}

Let $M$ be a two-dimensional simplicial complex.

(a) Let $\tau$ be an $i$-simplex $(1 \leq i \leq 2)$ and $\tilde{M}$ be obtained from $M$ by starring a vertex in $\tau$. If $M$ is a weak pseudomanifold, pseudomanifold or combinatorial 2-manifold then so is $\widetilde{M}$ with the same geometric carrier.

(b) Let $\tau_{1}, \tau_{2}$ are disjoint 2-simplices of $M$ and $u, v, w \notin V(M)$. Let $\widehat{M}:=\left(M \backslash\left\{\tau_{1}, \tau_{2}\right\}\right) \cup$ $\left(\left(\partial \tau_{1} \cup \partial \tau_{2}\right) * w\right)$. Let $N$ be the complex obtained from $M$ by starring $u$ in $\tau_{1}$ and $v$ in $\tau_{2}$. Let $\widetilde{N}$ be obtained from $N$ by identifying $u$ and $v$. If $M$ is a (weak) pseudomanifold then so is $\widetilde{N}$ and $\widetilde{N} \cong \widehat{M}$ (and hence $|\widehat{M}|$ is homeomorphic to the connected sum of $|M|$ and the pinched sphere whenever $M$ is a pseudomanifold).

\section{PROPOSITION 3.5}

Let $M_{1}$ and $M_{2}$ be two-dimensional weak pseudomanifolds.

(a) Let $\widetilde{M}_{j}$ be obtained from $M_{j}$ by starring a vertex on an $i$-simplex $(1 \leq i \leq 2) \sigma_{j}$ for $j=1,2$. If $\left(M_{1}, \sigma_{1}\right) \cong\left(M_{2}, \sigma_{2}\right)$, then $\widetilde{M}_{1} \cong \widetilde{M}_{2}$.

(b) If $\left(M_{1}, \sigma_{1}, \tau_{1}\right) \cong\left(M_{2}, \sigma_{2}, \tau_{2}\right)$ and $u_{1}, u_{2} \notin V\left(M_{1}\right) \cup V\left(M_{2}\right)$, where $\sigma_{j}, \tau_{j}$ are disjoint 2-simplices of $M_{j}$ for $j=1,2$, then $\left(M_{1} \backslash\left\{\sigma_{1}, \tau_{1}\right\}\right) \cup\left(\left(\partial \sigma_{1} \cup \partial \tau_{1}\right) * u_{1}\right) \cong$ $\left(M_{2} \backslash\left\{\sigma_{2}, \tau_{2}\right\}\right) \cup\left(\left(\partial \sigma_{2} \cup \partial \tau_{2}\right) * u_{2}\right)$.

\section{PROPOSITION 3.6}

Let $\varphi: K \rightarrow$ L be a simplicial map of degree $d>0$, where $K$ and $L$ are two-dimensional oriented pseudomanifolds. For a vertex $v$ of $L$, let $S_{v}:=\{\sigma \in K: \varphi(\sigma)$ is a 2-simplex containing $v\}$.

(a) If $\sigma$ is a 2-simplex of $L$ then $\varphi^{-1}(\sigma)$ contains at least $d$ simplices.

(b) If for some 2-simplex $\sigma$ of $L, \varphi^{-1}(\sigma)$ contains $d$ or $d+12$-simplices, say $\sigma_{1}, \ldots, \sigma_{d}$ $\left(\right.$ or $\left.\sigma_{d+1}\right)$, then $\sigma_{i}$ and $\sigma_{j}$ have at most one vertex in common for $i \neq j$.

(c) If for some vertex $v$ of degree $c$ of $L, S_{v}$ contains the 2-simplices $\tau_{i} \cup\left\{u_{i}\right\}, \tau_{i} \cup\left\{v_{i}\right\}$, where $\varphi\left(u_{i}\right)=\varphi\left(v_{i}\right)=v$ for $1 \leq i \leq p$, then $\#\left(S_{v}\right) \geq c d+2 p$.

Proof. (a) follows from the definition of the degree of a simplicial map.

If possible let $\varphi(u v x)=\varphi(u v y)=\sigma$. If $\varphi_{2}: C_{2}(K) \rightarrow C_{2}(L)$ is the homomorphism induced by $\varphi$ then, for any orientations of $K$ and $L, \varphi_{2}(+u v x)=-\varphi_{2}(+u v y)$ in $C_{2}(L)$. So, if $m$ is the coefficient of $+\sigma$ in $\varphi_{2}\left(C_{2}(K)\right)$ then $|m| \leq d+1-2$ and hence $\operatorname{deg}(\varphi) \leq d-1$, a contradiction. This proves (b).

By the same argument as in (b), $\varphi_{2}\left(+\tau_{i} \cup\left\{u_{i}\right\}\right)=-\varphi_{2}\left(+\tau_{i} \cup\left\{v_{i}\right\}\right)$, for $1 \leq i \leq p$. Therefore, for each 2-simplex $\sigma$ containing $v$ (as the degree of $\varphi$ is $d) \#\left(\varphi_{2}^{-1}(\sigma) \backslash\left\{\tau_{i} \cup\right.\right.$ $\left.\left.\left\{u_{i}\right\}, \tau_{i} \cup\left\{v_{i}\right\}: 1 \leq i \leq p\right\}\right) \geq d$. This proves (c).

For a simplicial map $\varphi: K \rightarrow L$, a $d$-simplex $\sigma$ is said to be collapsing if $\varphi(\sigma)$ is not a $d$-simplex. Let $\varphi: K \rightarrow S_{4}^{2}$ be a simplicial map, where $K$ is an $m$-vertex two-dimensional weak pseudomanifold and $S_{4}^{2}$ is the standard 2 -sphere (with 2 -simplices $\sigma_{1}, \ldots, \sigma_{4}$ ). The map $\varphi$ is said to be of type $\left(n_{1}, n_{2}, n_{3}, n_{4}\right)$ if $n_{i}$ is the number of triangles (in $K$ ) with image $\sigma_{i}(1 \leq i \leq 4)$ and $n_{1} \geq n_{2} \geq n_{3} \geq n_{4}$. 


\section{Proof of Theorem 1.1}

Throughout this section $M$ is an 8-vertex combinatorial 2-manifold.

The first lemma follows from the description of $R_{2}, R_{3}$ and $R_{4}$ in $\S 2$.

Lemma 4.1. If $R_{2}, R_{3}$ and $R_{4}$ are as in $\S 2$ then we have the following:

(i) $\left(R_{2}, v_{0} v_{1} v_{2}\right) \stackrel{\alpha_{1}}{\cong}\left(R_{2}, v_{0} v_{1} v_{3}\right) \stackrel{\alpha_{2}}{\cong}\left(R_{2}, v_{0} v_{2} v_{3}\right)$.

(ii) $\left(R_{2}, v_{1} v_{3} v_{5}\right) \stackrel{\alpha_{2}}{\cong}\left(R_{2}, v_{2} v_{3} v_{6}\right) \stackrel{\alpha_{3}}{\cong}\left(R_{2}, v_{1} v_{2} v_{4}\right)$.

(iii) $\left(R_{2}, \sigma\right) \cong\left(R_{2}, v_{3} v_{4} v_{5}\right)$, for $\sigma=v_{3} v_{4} v_{6}, v_{2} v_{5} v_{6}, v_{2} v_{4} v_{5}, v_{1} v_{4} v_{6}, v_{1} v_{5} v_{6}$.

(iv) $\left(R_{3}, v_{0} v_{1} v_{3}\right) \stackrel{\alpha_{2}}{\cong}\left(R_{3}, v_{0} v_{2} v_{3}\right) \stackrel{\alpha_{4}}{\cong}\left(R_{3}, v_{0} v_{2} v_{4}\right) \stackrel{\alpha_{2}}{\cong}\left(R_{3}, v_{0} v_{1} v_{4}\right)$.

(v) $\left(R_{3}, v_{1} v_{3} v_{5}\right) \stackrel{\alpha_{2}}{\cong}\left(R_{3}, v_{2} v_{3} v_{6}\right) \stackrel{\alpha_{4}}{\cong}\left(R_{3}, v_{2} v_{4} v_{5}\right) \stackrel{\alpha_{2}}{\cong}\left(R_{3}, v_{1} v_{4} v_{6}\right)$.

(vi) $\left(R_{3}, v_{3} v_{4} v_{5}\right) \stackrel{\alpha_{2}}{\cong}\left(R_{3}, v_{3} v_{4} v_{6}\right),\left(R_{3}, v_{1} v_{5} v_{6}\right) \stackrel{\alpha_{2}}{\cong}\left(R_{3}, v_{2} v_{5} v_{6}\right)$.

(vii) $\left(R_{4}, \sigma_{1}\right) \cong\left(R_{4}, \sigma_{2}\right)$ for any 2 triangles $\sigma_{1}, \sigma_{2}$ of $R_{4}$,

where $\alpha_{1}, \ldots, \alpha_{8}:\left\{v_{0}, \ldots, v_{6}\right\} \rightarrow\left\{v_{0}, \ldots, v_{6}\right\}$ are the permutations given by $\alpha_{1}=$ $\left(v_{2}, v_{3}\right)\left(v_{4}, v_{5}\right), \alpha_{2}=\left(v_{1}, v_{2}\right)\left(v_{5}, v_{6}\right), \alpha_{3}=\left(v_{1}, v_{3}\right)\left(v_{4}, v_{6}\right), \alpha_{4}=\left(v_{3}, v_{4}\right)\left(v_{5}, v_{6}\right), \alpha_{5}=$ $\left(v_{2}, v_{3}\right)\left(v_{0}, v_{6}\right), \alpha_{6}=\left(v_{2}, v_{4}\right)\left(v_{0}, v_{5}\right), \alpha_{7}=\left(v_{1}, v_{4}\right)\left(v_{0}, v_{6}\right)$ and $\alpha_{8}=\left(v_{1}, v_{3}\right)\left(v_{0}, v_{5}\right)$. Here $\left(R_{i}, \tau\right) \stackrel{\alpha_{l}}{\cong}\left(R_{j}, \sigma\right)$ means $\left(R_{i}, \tau\right)$ and $\left(R_{j}, \sigma\right)$ are isomorphic via the map $\alpha_{l}$.

Lemma 4.2. If $\chi(M)=1$ and $M$ has a vertex of degree 3 , then $M$ is isomorphic to $R_{5}$, $\ldots, R_{11}$ or $R_{12}$ defined above.

Proof. Let $v_{7}$ be a vertex of degree 3 of $M$ and let $\operatorname{Lk}\left(v_{7}\right)=C_{3}(a, b, c)$. Since $M \supsetneqq S_{4}^{2}$, $a b c$ is not a simplex. Let $\tilde{M}=\left(M \backslash\left\{v_{7}\right\}\right) \cup\{\tau\}$. Then $\tilde{M}$ is a 7-vertex combinatorial 2-manifold with $\chi(\tilde{M})=\chi(M)=1$. Hence, by Lemma $4.1,(\tilde{M}, \tau)$ is isomorphic to $\left(R_{2}, v_{0} v_{1} v_{2}\right),\left(R_{2}, v_{2} v_{3} v_{6}\right),\left(R_{2}, v_{1} v_{5} v_{6}\right),\left(R_{3}, v_{0} v_{1} v_{3}\right),\left(R_{3}, v_{2} v_{3} v_{6}\right),\left(R_{3}, v_{3} v_{4} v_{5}\right)$, $\left(R_{3}, v_{1} v_{5} v_{6}\right)$ or $\left(R_{4}, v_{0} v_{1} v_{3}\right)$.

If $(\widetilde{M}, \tau)$ is isomorphic to $\left(R_{2}, v_{0} v_{1} v_{2}\right)$ then, by Proposition $3.5(\mathrm{a}), M=(\tilde{M} \backslash\{\tau\}) \cup$ $\left(\partial \tau * v_{7}\right)$ is isomorphic to $R_{2,1}$, where $R_{2,1}=\left(R_{2} \backslash\left\{v_{0} v_{1} v_{2}\right\}\right) \cup\left(\left\{v_{0} v_{1}, v_{1} v_{2}, v_{0} v_{2}\right\} * v_{7}\right)$.

Since $R_{2,1}$ is $R_{5}, M$ is isomorphic to $R_{5}$.

Similarly in the other cases $M$ is isomorphic to one of the following: $R_{2,2}:=\left(R_{2} \backslash\right.$ $\left.\left\{v_{2} v_{3} v_{6}\right\}\right) \cup\left(\left\{v_{2} v_{3}, v_{3} v_{6}, v_{2} v_{6}\right\} * v_{7}\right), R_{2,3}:=\left(R_{2} \backslash\left\{v_{1} v_{5} v_{6}\right\}\right) \cup\left(\left\{v_{1} v_{5}, v_{5} v_{6}, v_{1} v_{6}\right\} *\right.$ $\left.v_{7}\right), R_{3,1}:=\left(R_{3} \backslash\left\{v_{0} v_{1} v_{3}\right\}\right) \cup\left(\left\{v_{0} v_{1}, v_{1} v_{3}, v_{0} v_{3}\right\} * v_{7}\right), R_{3,2}:=\left(R_{3} \backslash\left\{v_{2} v_{3} v_{6}\right\}\right) \cup$ $\left(\left\{v_{2} v_{3}, v_{3} v_{6}, v_{2} v_{6}\right\} * v_{7}\right), R_{3,3}:=\left(R_{3} \backslash\left\{v_{3} v_{4} v_{5}\right\}\right) \cup\left(\left\{v_{3} v_{4}, v_{4} v_{5}, v_{3} v_{5}\right\} * v_{7}\right), R_{3,4}:=\left(R_{3} \backslash\right.$ $\left.\left\{v_{1} v_{5} v_{6}\right\}\right) \cup\left(\left\{v_{1} v_{5}, v_{5} v_{6}, v_{1} v_{6}\right\} * v_{7}\right), R_{4,1}:=\left(R_{4} \backslash\left\{v_{0} v_{1} v_{3}\right\}\right) \cup\left(\left\{v_{0} v_{1}, v_{1} v_{3}, v_{0} v_{3}\right\} * v_{7}\right)$.

Now observe that $R_{2,2}=R_{6}, R_{2,3}=R_{7}, R_{3,1}=R_{8}, R_{3,2}=R_{9}, R_{3,3}=R_{10}, R_{3,4}=$ $R_{11}$ and $R_{4,1}=R_{12}$. This proves the lemma.

Lemma 4.3. If $\chi(M)=1$ and there exists a vertex of degree 7 of $M$ and no vertex of degree 3 , then $M$ is isomorphic to $R_{13}, \ldots, R_{16}$ or $R_{17}$.

Proof. By (1), the number of 2-simplices of $M$ is 14 . Let $v_{0}$ be a vertex of degree 7 and $\operatorname{Lk}\left(v_{0}\right)=C_{7}\left(v_{1}, \ldots, v_{7}\right)$.

Claim. Any 2-simplex not containing $v_{0}$ contains exactly one edge from $C_{7}\left(v_{1}, \ldots, v_{7}\right)$. 
Since the degree of each vertex is more than $3, v_{i} v_{i+1} v_{i+2}$ is not a simplex for $1 \leq i \leq 7$ (addition in the subscript is modulo 7). So, no 2-simplex contains more than one edge from $C_{7}\left(v_{1}, \ldots, v_{7}\right)$. Let $a b c$ be a simplex not containing $v_{0}$. If none of $a b, b c$ or $c a$ is from $C_{7}\left(v_{1}, \ldots, v_{7}\right)$ then the number of 2-simplices is more than 14 ( 7 through $v_{0}, 7$ through the edges in $C_{7}\left(v_{1}, \ldots, v_{7}\right)$ and $\left.a b c\right)$, a contradiction. These prove the claim.

First consider the case when there exists no triangle of the form $v_{i} v_{i+1} v_{i+3}$ or $v_{i} v_{i+1} v_{i+5}$. In this case, by the claim, the other triangles are $v_{1} v_{2} v_{5}, v_{2} v_{3} v_{6}, v_{3} v_{4} v_{7}, v_{1} v_{4} v_{5}, v_{2} v_{5} v_{6}$, $v_{3} v_{6} v_{7}$ and $v_{1} v_{4} v_{7}$. Then $M$ is $R_{17}$.

Now, assume that there exists a simplex of the form $v_{i} v_{i+1} v_{i+3}$ or $v_{i} v_{i+1} v_{i+5}$. We may assume that $v_{1} v_{2} v_{4}$ is a simplex (the case when $v_{1} v_{2} v_{6}$ is a simplex gives isomorphic complexes). Then, by repeated use of the claim, the following are the possibilities for the remaining 6 triangles.

(1) $v_{2} v_{4} v_{5}, \quad v_{2} v_{3} v_{5}, \quad v_{3} v_{5} v_{6}, \quad v_{3} v_{4} v_{6}, \quad v_{4} v_{6} v_{7}, \quad v_{1} v_{4} v_{7}$

(2) $v_{2} v_{4} v_{5}, \quad v_{2} v_{3} v_{5}, \quad v_{3} v_{5} v_{6}, \quad v_{3} v_{6} v_{7}, v_{1} v_{3} v_{7}, \quad v_{1} v_{3} v_{4}$

(3) $v_{2} v_{4} v_{5}, \quad v_{2} v_{3} v_{5}, \quad v_{3} v_{5} v_{6}, \quad v_{3} v_{6} v_{7}, v_{3} v_{4} v_{7}, v_{1} v_{4} v_{7}$,

(4) $v_{2} v_{4} v_{5}, \quad v_{2} v_{5} v_{6}, \quad v_{2} v_{3} v_{6}, \quad v_{3} v_{4} v_{6}, \quad v_{4} v_{6} v_{7}, \quad v_{1} v_{4} v_{7}$

(5) $v_{2} v_{4} v_{5}, \quad v_{2} v_{5} v_{6}, \quad v_{2} v_{3} v_{6}, \quad v_{3} v_{6} v_{7}, v_{3} v_{4} v_{7}, \quad v_{1} v_{4} v_{7}$

(6) $v_{2} v_{4} v_{5}, \quad v_{2} v_{5} v_{6}, \quad v_{2} v_{3} v_{6}, \quad v_{3} v_{6} v_{7}, v_{1} v_{3} v_{7}, \quad v_{1} v_{3} v_{4}$,

(7) $v_{2} v_{4} v_{5}, \quad v_{2} v_{5} v_{6}, \quad v_{2} v_{6} v_{7}, \quad v_{2} v_{3} v_{7}, \quad v_{3} v_{4} v_{7}, \quad v_{1} v_{4} v_{7}$

(8) $v_{2} v_{4} v_{5}, \quad v_{2} v_{5} v_{6}, \quad v_{2} v_{6} v_{7}, \quad v_{2} v_{3} v_{7}, \quad v_{1} v_{3} v_{7}, \quad v_{1} v_{3} v_{4}$.

For $1 \leq i \leq 8$, let $M_{i}$ be the combinatorial 2-manifold arising in case (i) above. Let $\alpha_{1}, \alpha_{2}, \alpha_{3}:\left\{v_{0}, \ldots, v_{7}\right\} \rightarrow\left\{v_{0}, \ldots, v_{7}\right\}$ be the permutations given by $\alpha_{1}=\left(v_{1}, v_{6}\right)$ $\left(v_{2}, v_{5}\right)\left(v_{3}, v_{4}\right), \alpha_{2}=\left(v_{1}, v_{5}\right)\left(v_{2}, v_{4}\right)\left(v_{6}, v_{7}\right)$ and $\alpha_{3}=\left(v_{1}, v_{4}\right)\left(v_{2}, v_{3}\right)\left(v_{5}, v_{7}\right)$. Then

$$
M_{8} \stackrel{\alpha_{3}}{\cong} M_{2} \stackrel{\alpha_{1}}{\cong} M_{1}=R_{13}, \quad M_{6} \stackrel{\alpha_{2}}{\cong} M_{3}=R_{14}, \quad M_{7} \stackrel{\alpha_{2}}{\cong} M_{4}=R_{15}, \quad M_{5}=R_{16} .
$$

So, in this case, $M$ is isomorphic to $R_{13}, R_{14}, R_{15}$ or $R_{16}$. This proves the lemma.

Lemma 4.4. If $\chi(M)=1$ and $3<\operatorname{deg}(v)<7$, for each vertex $v$, then $M$ is isomorphic to $R_{18}, R_{19}$ or $R_{20}$.

Proof. Since (by (1)) the number of edges of $M$ is 21 , there exists a vertex (say $v_{0}$ ) of degree 6. Let $\operatorname{Lk}\left(v_{0}\right)=C_{6}\left(v_{1}, \ldots, v_{6}\right)$. Let $v_{7}$ be the remaining vertex. If $\operatorname{Lk}\left(v_{0}\right)$ and $\operatorname{Lk}\left(v_{7}\right)$ have no common edge then number of 2-simplices is more than 14 (6 through $v_{0}, 6$ more through the edges in $C_{6}\left(v_{1}, \ldots, v_{6}\right)$ and at least 4 more through $\left.v_{7}\right)$, a contradiction. Without loss of generality, we can assume that $v_{4} v_{5} v_{7}$ is a simplex.

Let $v v_{1} v_{2}\left(\neq v_{0} v_{1} v_{2}\right)$ be the other simplex containing $v_{1} v_{2}$. Since the degree of each vertex is more than $3, v \notin\left\{v_{3}, v_{6}\right\}$. Hence, without loss of generality, we can assume that $v=v_{4}$ or $v_{7}$.

Case I. $v_{1} v_{2} v_{4}$ is a simplex. As $v_{2} v_{3} v_{4}$ is not a simplex, $\operatorname{Lk}\left(v_{4}\right)=C_{6}\left(v_{3}, v_{0}, v_{5}, v_{7}, v_{2}, v_{1}\right)$. Since $v_{1} v_{5} v_{6}$ is not a simplex, $\operatorname{Lk}\left(v_{1}\right)$ is $C_{5}\left(v_{6}, v_{0}, v_{2}, v_{4}, v_{3}\right)$ or $C_{6}\left(v_{6}, v_{0}, v_{2}, v_{4}, v_{3}, v_{7}\right)$.

Subcase I.1. $\operatorname{Lk}\left(v_{1}\right)=C_{5}\left(v_{6}, v_{0}, v_{2}, v_{4}, v_{3}\right)$. Since $\operatorname{deg}\left(v_{7}\right)>3, v_{2} v_{5} \notin \operatorname{Lk}\left(v_{7}\right)$. Therefore $v_{2} v_{3}$ or $v_{2} v_{6}$ is in $\operatorname{Lk}\left(v_{7}\right)$.

In the first case, from the links of $v_{3}$ and $v_{7}, v_{3} v_{6} v_{7}$ and $v_{5} v_{6} v_{7}$ are simplices. Then $M$ is isomorphic, via the map $\left(v_{0}, v_{6}, v_{2}, v_{1}, v_{5}, v_{7}\right)\left(v_{3}, v_{4}\right)$, to $R_{20}$. 
In the second case, $v_{2} v_{3} v_{6}$ is a simplex through $v_{2}$ and hence $v_{5} v_{6} v_{7}$ is a simplex. Then $M$ is isomorphic, via the map $\left(v_{0}, v_{6}, v_{3}, v_{5}, v_{7}\right)\left(v_{1}, v_{4}, v_{2}\right)$, to $R_{19}$.

Subcase I.2. $\operatorname{Lk}\left(v_{1}\right)=C_{6}\left(v_{6}, v_{0}, v_{2}, v_{4}, v_{3}, v_{7}\right)$. Then $\operatorname{Lk}\left(v_{7}\right)$ is $C_{6}\left(v_{2}, v_{4}, v_{5}, v_{3}, v_{1}, v_{6}\right)$ or $C_{6}\left(v_{2}, v_{4}, v_{5}, v_{6}, v_{1}, v_{3}\right)$. If $\operatorname{Lk}\left(v_{7}\right)=C_{6}\left(v_{2}, v_{4}, v_{5}, v_{3}, v_{1}, v_{6}\right)$ then $\operatorname{Lk}\left(v_{2}\right)=C_{6}\left(v_{1}\right.$, $\left.v_{0}, v_{3}, v_{6}, v_{7}, v_{4}\right)$. These give more than 14 triangles, which is not possible. $\operatorname{So} \operatorname{Lk}\left(v_{7}\right)=$ $C_{6}\left(v_{2}, v_{4}, v_{5}, v_{6}, v_{1}, v_{3}\right)$. Then $M$ is isomorphic, via the map $\left(v_{0}, v_{2}, v_{5}\right)\left(v_{7}, v_{3}, v_{4}, v_{1}, v_{6}\right)$ to $R_{19}$.

Case II. $v_{1} v_{2} v_{7}$ is a simplex. Since the degree of each vertex is more than 3 , the 2-simplex $\left(\neq v_{0} v_{3} v_{4}\right)$ containing $v_{3} v_{4}$ is $v_{1} v_{3} v_{4}, v_{3} v_{4} v_{6}$ or $v_{3} v_{4} v_{7}$.

In the first case, by similar argument as above, $M$ is isomorphic to $R_{18}$ or $R_{20}$.

In each of the other two cases, $M$ is isomorphic to $R_{18}, R_{19}$ or $R_{20}$. This completes the proof of Lemma 4.4 .

Lemma 4.5. If $\chi(M)=0$ and there exists a vertex of degree 3 in $M$, then $M$ is isomorphic to $T_{3}$.

Proof. Let $\underset{\sim}{\operatorname{deg}}\left(v_{0}\right)=3$ and let $\operatorname{Lk}\left(v_{0}\right)=C_{3}\left(v_{1}, v_{2}, v_{3}\right)$. Since $M ¥ S_{4}^{2}, v_{1} v_{2} v_{3}$ is not a simplex. Let $\widetilde{M}$ be the complex on the vertex set $V(M) \backslash\left\{v_{0}\right\}$ and whose 2-simplices are $v_{1} v_{2} v_{3}$ and those of $M$ which do not contain $v_{0}$. Then $\widetilde{M}$ is a 7 vertex 2-manifold which has 14 triangles and hence 21 edges. From Proposition 3.1, $\widetilde{M}$ is isomorphic to $T_{1}$. Then, by Proposition 3.5(a), $M$ is isomorphic to $T_{3}$.

Lemma 4.6. If $\chi(M)=0$ and $M$ has no vertex of degree 7 , then $M$ is isomorphic to $T_{2}$.

Proof. Since the degree of each vertex is less than 7 , the degree of each vertex has to be 6 . Let $v_{0}$ be a vertex whose link is $C_{6}\left(v_{1}, \ldots, v_{6}\right)$. Let $v_{7}$ be the remaining vertex. Then $v_{1}, \ldots, v_{6} \in \operatorname{Lk}\left(v_{7}\right)$. Since the degree of each vertex is more than $3, v_{i} v_{i+1} v_{i+2}$ is not a simplex in $M$ for $1 \leq i \leq 6$ (addition in the subscripts are modulo 6). Since $|M|$ is not homeomorphic to $S^{2}, \operatorname{Lk}\left(v_{7}\right) \neq C_{6}\left(v_{1}, \ldots, v_{6}\right)$. Without loss of generality, assume $v_{1} v_{2} \notin \operatorname{Lk}\left(v_{7}\right)$. Then either $v_{1} v_{2} v_{4}$ or $v_{1} v_{2} v_{5}$ is the other 2 -simplex containing $v_{1} v_{2}$. Assume, without loss of generality, that $v_{1} v_{2} v_{4}$ is a simplex. So, $v_{3} v_{0}, v_{0} v_{5}$ and $v_{1} v_{2}$ are edges in the link of $v_{4}$.

Claim. Neither $v_{1} v_{3}$ nor $v_{2} v_{5}$ is an edge in the link of $v_{4}$.

If $v_{2} v_{5} \in \operatorname{Lk}\left(v_{4}\right)$ then $\operatorname{Lk}\left(v_{4}\right)=C_{6}\left(v_{3}, v_{0}, v_{5}, v_{2}, v_{1}, v_{7}\right)$. Since $v_{1} v_{5} v_{6}$ is not a simplex, $\operatorname{Lk}\left(v_{1}\right)=C_{6}\left(v_{6}, v_{0}, v_{2}, v_{4}, v_{7}, v_{3}\right)$ and hence $\operatorname{Lk}\left(v_{3}\right)=C_{6}\left(v_{2}, v_{0}, v_{4}, v_{7}, v_{1}, v_{6}\right)$. These imply $\operatorname{deg}\left(v_{2}\right)=7$, a contradiction.

If $v_{1} v_{3} \in \operatorname{Lk}\left(v_{4}\right)$ then $\operatorname{Lk}\left(v_{1}\right)=C_{6}\left(v_{6}, v_{0}, v_{2}, v_{4}, v_{3}, v_{7}\right), \operatorname{Lk}\left(v_{4}\right)=C_{6}\left(v_{3}, v_{0}, v_{5}, v_{7}\right.$, $\left.v_{2}, v_{1}\right)$ and $\operatorname{Lk}\left(v_{3}\right)=C_{6}\left(v_{2}, v_{0}, v_{4}, v_{1}, v_{7}, v\right)$, where $v=v_{5}$ or $v_{6}$. If $v=v_{5}$ then, $\operatorname{Lk}\left(v_{5}\right)=C_{6}\left(v_{4}, v_{0}, v_{6}, v_{2}, v_{3}, v_{7}\right)$. These imply, $v_{0} v_{2}, v_{1} v_{2}, v_{3} v_{2}, \ldots, v_{7} v_{2}$ are edges in $M$ and hence, $\operatorname{deg}\left(v_{2}\right)=7$, a contradiction. If $v=v_{6}$ then $C_{3}\left(v_{1}, v_{3}, v_{6}\right) \subseteq \operatorname{Lk}\left(v_{7}\right)$, a contradiction. This proves the claim.

Since $v_{2} v_{3} v_{4}$ is not a simplex, by the claim, $\operatorname{Lk}\left(v_{4}\right)=C_{6}\left(v_{3}, v_{0}, v_{5}, v_{1}, v_{2}, v_{7}\right)$ and $\operatorname{Lk}\left(v_{1}\right)=C_{6}\left(v_{6}, v_{0}, v_{2}, v_{4}, v_{5}, v_{7}\right)$. Then $\operatorname{Lk}\left(v_{5}\right)=C_{6}\left(v_{6}, v_{0}, v_{4}, v_{1}, v_{7}, y\right)$, where $y=$ $v_{2}$ or $v_{3}$. If $y=v_{2}$ then $\operatorname{deg}\left(v_{2}\right)=7$, a contradiction. So, $y=v_{3}$. These imply $\operatorname{Lk}\left(v_{2}\right)=$ $C_{6}\left(v_{1}, v_{0}, v_{3}, v_{6}, v_{7}, v_{4}\right)$. Then $M$ is isomorphic, via the map $\left(v_{1}, v_{4}, v_{5}, v_{3}, v_{7}\right)\left(v_{2}, v_{6}\right)$, to $T_{2}$. 
Lemma 4.7. If $\chi(M)=0$ and there exists a vertex of degree 7 in $M$ and no vertex of degree 3 , then $M$ is isomorphic to $T_{4}, \ldots, T_{8}, K_{1}, \ldots, K_{5}$ or $K_{6}$.

Proof. Let $\operatorname{deg}\left(v_{0}\right)=7$ and let $\operatorname{Lk}\left(v_{0}\right)=C_{7}\left(v_{1}, \ldots, v_{7}\right)$. Since the degree of each vertex is more than 3 and $M \neq R_{17}$, there exists $i \in\{1, \ldots, 7\}$ such that $v_{i} v_{i+1} v_{i+3}$ or $v_{i} v_{i+1} v_{i+5}$ is a simplex (additions in the subscripts are modulo 7). We may assume that $v_{1} v_{2} v_{4}$ is a simplex (cases when $v_{1} v_{2} v_{6}$ is a simplex give isomorphic complexes). Then the 2-simplex ( $\neq v_{0} v_{1} v_{7}$ ) containing $v_{1} v_{7}$ is $v_{1} v_{7} v_{3}, v_{1} v_{7} v_{4}$ or $v_{1} v_{7} v_{5}$.

Case I. $v_{1} v_{4} v_{7}$ is a simplex. If $v_{4} v_{7} v_{6}$ is a simplex then $\operatorname{Lk}\left(v_{7}\right)=C_{4}\left(v_{1}, v_{0}, v_{6}, v_{4}\right)$, $\operatorname{Lk}\left(v_{4}\right)=C_{7}\left(v_{3}, v_{0}, v_{5}, v_{2}, v_{1}, v_{7}, v_{6}\right)$ and $\operatorname{Lk}\left(v_{1}\right)=C_{4}\left(v_{2}, v_{0}, v_{7}, v_{4}\right)$. Now, $\operatorname{Lk}\left(v_{2}\right)$ is $C_{5}\left(v_{3}, v_{0}, v_{1}, v_{4}, v_{5}\right)$ or $C_{6}\left(v_{3}, v_{0}, v_{1}, v_{4}, v_{5}, v_{6}\right)$. In the first case, $\operatorname{Lk}\left(v_{3}\right)=C_{5}\left(v_{2}, v_{0}\right.$, $\left.v_{4}, v_{6}, v_{5}\right)$. Then the links of the remaining vertices are complete and the degree sequence of $M$ is $7^{2} \cdot 5^{4} \cdot 4^{2}$ and hence $\chi(M)=-1$, a contradiction. In the second case, the links of the remaining vertices are complete and the degree sequence of $M$ is $7^{2} \cdot 6^{2} \cdot 4^{4}$, a contradiction again. So, the 2 -simplex $\left(\neq v_{0} v_{4} v_{7}\right)$ containing $v_{4} v_{7}$ is $v_{3} v_{4} v_{7}$ or $v_{4} v_{5} v_{7}$.

Subcase I.1. $v_{3} v_{4} v_{7}$ is a simplex. Since $v_{4} v_{5} v_{6}$ is not a simplex, $\operatorname{Lk}\left(v_{4}\right)=C_{6}\left(v_{3}, v_{0}, v_{5}\right.$, $\left.v_{2}, v_{1}, v_{7}\right)$ and hence $\operatorname{Lk}\left(v_{1}\right)=C_{4}\left(v_{7}, v_{0}, v_{2}, v_{4}\right)$. Then the edge $v_{5} v_{6}\left(\operatorname{of} \operatorname{Lk}\left(v_{0}\right)\right)$ is either in $\operatorname{Lk}\left(v_{2}\right)$ or in $\operatorname{Lk}\left(v_{3}\right)$.

If $v_{5} v_{6}$ is in $\operatorname{Lk}\left(v_{2}\right)$, then $\operatorname{Lk}\left(v_{2}\right)$ is $C_{7}\left(v_{3}, v_{0}, v_{1}, v_{4}, v_{5}, v_{6}, v_{7}\right)$ or $C_{6}\left(v_{3}, v_{0}, v_{1}, v_{4}, v_{5}\right.$, $\left.v_{6}\right)$. In the first case, the links of the remaining vertices are complete. These imply that $f_{2}(M)=14$ and hence $\chi(M)=-1$, a contradiction. In the second case, $\operatorname{Lk}\left(v_{6}\right)=C_{5}\left(v_{7}\right.$, $\left.v_{0}, v_{5}, v_{2}, v_{3}\right)$. Then no more 2 -simplices are possible. These imply that $f_{2}(M)=14$, a contradiction again. So, $v_{5} v_{6}$ is in $\operatorname{Lk}\left(v_{3}\right)$.

If $v_{2} v_{3} v_{5}$ is a simplex then $\operatorname{Lk}\left(v_{3}\right)=C_{6}\left(v_{2}, v_{0}, v_{4}, v_{7}, v_{6}, v_{5}\right)$. These complete all the links of the remaining vertices. In this case, $f_{2}(M)=14$, a contradiction. Hence, the 2simplex $\left(\neq v_{0} v_{2} v_{3}\right.$ ) containing $v_{2} v_{3}$ is $v_{2} v_{3} v_{6}$. Then $\operatorname{Lk}\left(v_{3}\right)=C_{6}\left(v_{2}, v_{0}, v_{4}, v_{7}, v_{5}, v_{6}\right)$. Now, $\operatorname{Lk}\left(v_{2}\right)$ and $\operatorname{Lk}\left(v_{7}\right)$ show that the remaining two simplices are $v_{2} v_{6} v_{7}$ and $v_{2} v_{5} v_{7}$. Then $M$ is isomorphic, via the map $\left(v_{0}, v_{4}, v_{2}\right)\left(v_{1}, v_{3}, v_{5}\right)$, to $K_{3}$.

Subcase I.2. $v_{4} v_{5} v_{7}$ is a simplex. To complete $\operatorname{Lk}\left(v_{4}\right), v_{2} v_{4} v_{6}$ and $v_{3} v_{4} v_{6}$ have to be simplices. Then the other triangle containing $v_{5} v_{6}$ is either $v_{2} v_{5} v_{6} v_{3} v_{5} v_{6}$.

In the first case, using the similar method as above, $M$ is isomorphic to $T_{4}$ or $T_{7}$.

In the second case, $M$ is isomorphic to $K_{3}$.

Case II. $v_{1} v_{3} v_{7}$ is a simplex. Since $v_{6} v_{7}$ is an edge, one of $v_{2} v_{6} v_{7}, v_{3} v_{6} v_{7}$ or $v_{4} v_{6} v_{7}$ is a triangle.

In the first case, $M$ is isomorphic to $K_{1}, \ldots, K_{4}, T_{4}$ or $T_{5}$.

In the second case, $M$ is isomorphic to $T_{5}, T_{6}, T_{7}, K_{1}, \ldots, K_{4}$ or $K_{6}$.

In the third case, $M$ is isomorphic to $T_{4}$ or $K_{3}$.

Case III. $v_{1} v_{5} v_{7}$ is a simplex. Then one of $v_{1} v_{5} v_{6}, v_{2} v_{5} v_{6}$ or $v_{3} v_{5} v_{6}$ is a triangle containing $v_{5} v_{6}$.

In the first case, $M$ is isomorphic to $K_{1}, \ldots, K_{6}$.

In the second case, $M$ is isomorphic to $T_{5}, \ldots, T_{8}, K_{2}, K_{3}$ or $K_{4}$,

In the third case, $M$ is isomorphic to $K_{1}, \ldots, K_{5}, T_{5}$ or $T_{8}$. This completes the proof of Lemma 4.7.

Lemma 4.8. The combinatorial 2-manifolds mentioned in Theorem 1.1 are pairwise nonisomorphic. 
Proof. For $2 \leq i<j \leq 8, T_{i} \cong T_{j}$ implies that the degree sequence of $T_{i}$ is the same as the degree sequence of $T_{j}$. This implies that $(i, j)=(5,8)$. If $\varphi$ is an isomorphism from $T_{5}$ to $T_{8}$, then $\varphi\left(v_{1} v_{2}\right)=v_{2} v_{5}$ ( since $\operatorname{deg}_{T_{5}}\left(v_{j}\right)=\operatorname{deg}_{T_{8}}\left(\varphi\left(v_{j}\right)\right)$. This implies $\varphi\left(v_{1} v_{2} v_{4}\right)=$ $v_{2} v_{5} v_{0}$ or $v_{2} v_{5} v_{3}$. Then $\varphi\left(v_{4}\right)=v_{0}$ or $v_{3}$. But $\operatorname{deg}_{T_{5}}\left(v_{4}\right)=6$ and $\operatorname{deg}_{T_{8}}\left(v_{0}\right)=5=$ $\operatorname{deg}_{T_{8}}\left(v_{3}\right)$, a contradiction. So, $T_{i} ¥ T_{j}$ for $2 \leq i \neq j \leq 8$.

Similarly, for $1 \leq i<j \leq 6, K_{i} \cong K_{j}$ implies that the degree sequence of $K_{i}$ is the same as the degree sequence of $K_{j}$ and hence $(i, j)=(2,4)$. If $\alpha$ is an isomorphism from $K_{2}$ to $K_{4}$, then $\alpha\left(\left\{v_{0}, v_{4}, v_{5}\right\}\right)=\left\{v_{1}, v_{4}, v_{5}\right\}$ (since $\alpha$ takes vertices of degree 7 in $K_{2}$ to vertices of degree 7 of $K_{4}$ ). Now, $v_{0} v_{4} v_{5} \in K_{2}$ whereas $v_{1} v_{4} v_{5} \notin K_{4}$. Hence, $K_{2} \nsucceq K_{4}$. So, $K_{i} \neq K_{j}$ for $1 \leq i \neq j \leq 6$.

Repeating the above argument we find that for $5 \leq i<j \leq 20, R_{i} \cong R_{j}$ implies $(i, j)=(5,10),(14,16)$ or $(18,19)$.

If possible, let $\beta_{1}: R_{5} \rightarrow R_{10}$ be an isomorphism. Since $\operatorname{deg}\left(\beta_{1}(v)\right)=\operatorname{deg}(v)$ for each vertex $v$ in $R_{5}, \beta_{1}\left(v_{7}\right)=v_{7}$ and $\beta_{1}\left(v_{0}\right)=v_{0}$. Now, $v_{0} v_{7}$ is an edge in $R_{5}$ whereas $v_{0} v_{7}$ is not an edge in $R_{10}$. So, $R_{5} ¥ R_{10}$.

If possible, let $\beta_{2}: R_{14} \rightarrow R_{16}$ be an isomorphism. Since $\operatorname{deg}\left(\beta_{2}(v)\right)=\operatorname{deg}(v)$ for each vertex $v$ in $R_{14}, \beta_{2}\left(v_{0}\right)=v_{0}$ and $\beta_{1}\left(\left\{v_{3}, v_{4}\right\}\right)=\left\{v_{2}, v_{4}\right\}$. Now, $v_{0} v_{3} v_{4}$ is a simplex in $R_{14}$ whereas $v_{0} v_{2} v_{4}$ is not a simplex in $R_{16}$. Thus, $R_{14} ¥ R_{16}$.

In $R_{19}$, both the degree 4 vertices form an edge but that is not the case in $R_{18}$. Thus, $R_{18} \not R_{19}$. So, $R_{i} \not R_{j}$ for $5 \leq i \neq j \leq 20$.

If, for $10 \leq i<j \leq 23, S_{i} \cong S_{j}$ then the degree sequence of $S_{i}$ is equal to the degree sequence of $S_{j}$. This implies that $i=16$ and $j=18$. Now, if $\gamma$ is an isomorphism between $S_{16}$ and $S_{18}$, then $\gamma$ takes vertices of degree 3 in $S_{16}$ to vertices of degree 3 of $S_{18}$. Observe that the links of the vertices of degree 3 of $S_{16}$ have a vertex of degree 4 whereas those of $S_{18}$ have no vertex of degree 4 . This shows that such a $\gamma$ does not exist. Hence, $S_{i} ¥ S_{j}$ for $10 \leq i \neq j \leq 23$.

The lemma now follows from the fact that $S_{i}(10 \leq i \leq 23)$ triangulates $S^{2}, R_{j}$ $(5 \leq j \leq 20)$ triangulates $\mathbb{R} P^{2}, T_{k}(2 \leq k \leq 8)$ triangulates $S^{1} \times S^{1}, K_{p}(1 \leq p \leq 6)$ triangulates the Klein bottle and $|D|$ is disconnected.

Proof of Theorem 1.1. Let $M$ be an 8-vertex combinatorial 2-manifold. Then $\frac{3 \times 8}{2} \leq$ $f_{1}(M) \leq\left(\begin{array}{l}8 \\ 2\end{array}\right), \chi(M)=8-f_{1}(M)+f_{2}(M)$ and $3 f_{2}(M)=2 f_{1}(M)$. This implies $12 \leq f_{1}(M) \leq 27$ and hence $-1 \leq \chi(M) \leq 4$. But it is known (e.g., see [7,9]) that there does not exist any 8-vertex combinatorial 2-manifold $M$ with $\chi(M)=-1$. If $\chi(M) \geq 3$ then $|M|$ is disconnected and since the number of vertices is $8, M$ is isomorphic to $D$. So, if $M \neq D$ then $0 \leq \chi(M) \leq 2$. The theorem now follows from Lemmas $4.2, \ldots, 4.8$ and Proposition 3.2.

\section{Proof of Theorem 1.2}

Throughout this section $M$ is an 8-vertex two-dimensional weak pseudomanifold which is not a combinatorial 2-manifold.

Lemma 5.1. If $M$ has a vertex, say $v_{0}$, whose link is of the form $C_{3}\left(v_{1}, v_{2}, v_{3}\right) \sqcup$ $C_{4}\left(v_{4}, v_{5}, v_{6}, v_{7}\right)$, where $v_{1} v_{2} v_{3}$ is a simplex, then $M$ is isomorphic to $Q_{3}, Q_{5}, Q_{6}, Q_{12}$ or $Q_{16}$. 
Proof. Here we have two cases: (I) At least one of $v_{4} v_{6}$ or $v_{5} v_{7}$ is an edge of $M$ and (II) Neither $v_{4} v_{6}$ nor $v_{5} v_{7}$ is an edge of $M$.

Case I. We can assume, without loss of generality, that $v_{4} v_{6}$ is an edge of $M$.

Subcase I.1. The triangles through $v_{4} v_{6}$ are $v_{4} v_{5} v_{6}$ and $v_{4} v_{6} v_{7}$. Then, $M$ is $Q_{3}$.

Subcase I.2. Exactly one of $v_{4} v_{5} v_{6}$ or $v_{4} v_{6} v_{7}$ is a simplex. Assume, without loss of generality, that $v_{4} v_{5} v_{6}$ is a simplex. Then, the other triangle through $v_{4} v_{6}$ must be one of $v_{1} v_{4} v_{6}$, $v_{2} v_{4} v_{6}$ or $v_{3} v_{4} v_{6}$. Assume, without loss of generality, that $v_{1} v_{4} v_{6}$ is a simplex in $M$. Then $v_{1} v_{6} v_{7}$ and $v_{1} v_{4} v_{7}$ have to be simplices. Here $M$ is $Q_{5}$.

Subcase I.3. Neither $v_{4} v_{5} v_{6}$ nor $v_{4} v_{6} v_{7}$ is a simplex of $M$. The triangles through $v_{4} v_{6}$ are of the form $v_{1} v_{4} v_{6}, v_{2} v_{4} v_{6}$ or $v_{3} v_{4} v_{6}$. Assume, without loss of generality, that $v_{1} v_{4} v_{6}$ and $v_{2} v_{4} v_{6}$ are simplices of $M$. The triangle $\left(\neq v_{1} v_{4} v_{6}\right)$ having $v_{1} v_{4}$ as an edge is either $v_{1} v_{4} v_{5}$ or $v_{1} v_{4} v_{7}$. We can assume, without loss of generality, that $v_{1} v_{4} v_{5}$ is a simplex. Then, the other triangle through $v_{1} v_{5}$ is $v_{1} v_{5} v_{6}$ or $v_{1} v_{5} v_{7}$.

In the first case (by considering $\operatorname{Lk}\left(v_{6}\right)$ and $\left.\operatorname{Lk}\left(v_{4}\right)\right), v_{2} v_{6} v_{7}$ and $v_{2} v_{4} v_{7}$ are simplices. Here $M$ is $Q_{12}$.

In the second case, $\operatorname{Lk}\left(v_{4}\right)=C_{6}\left(v_{5}, v_{0}, v_{7}, v_{2}, v_{6}, v_{1}\right)$ and $\operatorname{Lk}\left(v_{1}\right)=C_{3}\left(v_{0}, v_{2}, v_{3}\right) \sqcup$ $C_{4}\left(v_{6}, v_{4}, v_{5}, v_{7}\right)$ and hence $\operatorname{Lk}\left(v_{6}\right)=C_{6}\left(v_{5}, v_{0}, v_{7}, v_{1}, v_{4}, v_{2}\right)$. The link of $v_{7}$ shows that $v_{2} v_{5} v_{7}$ is a simplex. Then $M$ is $Q_{16}$.

Case II. Assume, without loss of generality, that the second 2-simplex containing $v_{4} v_{5}$ is $v_{1} v_{4} v_{5}$. Then (since, $v_{4} v_{6}$ and $v_{5} v_{7}$ are non-edges), $\operatorname{Lk}\left(v_{1}\right)=C_{3}\left(v_{0}, v_{2}, v_{3}\right) \sqcup C_{4}\left(v_{4}\right.$, $\left.v_{5}, v_{6}, v_{7}\right)$. In this case, $M$ is $Q_{6}$.

Lemma 5.2. If $M$ has a vertex, say $v_{0}$, whose link is of the form $C_{3}\left(v_{1}, v_{2}, v_{3}\right) \sqcup$ $C_{4}\left(v_{4}, v_{5}, v_{6}, v_{7}\right)$, where $v_{4} v_{6}$ and $v_{5} v_{7}$ are edges but $v_{1} v_{2} v_{3}$ is not a simplex, then $M$ is isomorphic to $P_{29}, \ldots, P_{32}, P_{35}, P_{37}, P_{38}, Q_{11}$ or $Q_{14}$.

Proof. We have three cases: (I) Both $v_{4} v_{5} v_{6}$ and $v_{4} v_{6} v_{7}$ are simplices, (II) exactly one of $v_{4} v_{5} v_{6}$ and $v_{4} v_{6} v_{7}$ is a simplex and (III) neither $v_{4} v_{5} v_{6}$ nor $v_{4} v_{6} v_{7}$ is a simplex.

Case I. The triangles through $v_{5} v_{7}$ are of the form $v_{1} v_{5} v_{7}, v_{2} v_{5} v_{7}$ or $v_{3} v_{5} v_{7}$. We can assume, without loss of generality, that $v_{1} v_{5} v_{7}$ and $v_{2} v_{5} v_{7}$ are in $M$. Consider the triangle through $v_{1} v_{5}$ other than $v_{1} v_{5} v_{7}$. Clearly, it is either $v_{1} v_{2} v_{5}$ or $v_{1} v_{3} v_{5}$.

In the former case, to complete $\operatorname{Lk}\left(v_{1}\right)$ and $\operatorname{Lk}\left(v_{3}\right), v_{1} v_{3} v_{7}$ and $v_{2} v_{3} v_{7}$ have to be simplices. Here $M$ is $Q_{11}$.

In the latter case, $v_{2} v_{3} v_{5}$ has to be a simplex. This implies $v_{1} v_{2} v_{7}$ is also a simplex. Then $M$ is isomorphic, via the map $\left(v_{5}, v_{7}\right)$, to $Q_{11}$.

Case II. Assume, without loss of generality, that $v_{4} v_{5} v_{6}$ is a simplex. Then, the other triangle through $v_{4} v_{6}$ must be one of $v_{1} v_{4} v_{6}, v_{2} v_{4} v_{6}$ or $v_{3} v_{4} v_{6}$. Assume, without loss of generality, that $v_{1} v_{4} v_{6}$ is in $M$. The triangles through $v_{5} v_{7}$ are of the form $v_{1} v_{5} v_{7}, v_{2} v_{5} v_{7}$ or $v_{3} v_{5} v_{7}$. Without loss of generality we can assume that either $v_{1} v_{5} v_{7}$ and $v_{2} v_{5} v_{7}$ are in $M$ or $v_{2} v_{5} v_{7}$ and $v_{3} v_{5} v_{7}$ are in $M$.

In the first case, $M$ is $P_{29}, P_{30}$ or $P_{31}$.

In the second case, $M$ is isomorphic to $P_{32}, P_{35}$ or $Q_{14}$.

Case III. Without loss of generality, assume that the triangles through $v_{4} v_{6}$ are $v_{1} v_{4} v_{6}$ and $v_{2} v_{4} v_{6}$. Then assume, without loss of generality, that the triangles through $v_{5} v_{7}$ are either $v_{1} v_{5} v_{7}$ and $v_{2} v_{5} v_{7}$ or $v_{2} v_{5} v_{7}$ and $v_{3} v_{5} v_{7}$. 
In the first case, $M$ is isomorphic to $P_{37}$.

In the second case, $M$ is isomorphic to $P_{38}$.

Lemma 5.3. If there exists a vertex, say $v_{0}$, whose link in $M$ is of the form $C_{3}\left(v_{1}, v_{2}\right.$, $\left.v_{3}\right) \sqcup C_{4}\left(v_{4}, v_{5}, v_{6}, v_{7}\right)$, where $v_{1} v_{2} v_{3}$ and $v_{4} v_{6}$ are not simplices, then $M$ is isomorphic to $P_{3}, P_{4}, P_{6}, P_{9}, P_{11}, P_{13}, P_{15}, P_{22}, P_{23}, P_{25}, P_{28}, P_{30}, P_{34}, Q_{7}, Q_{8}$ or $Q_{11}$.

Proof. The complex $\left.\tilde{M}=\left(M \backslash\left\{v_{0}\right\}\right) \cup\left\{v_{1} v_{2} v_{3}, v_{4} v_{5} v_{6}, v_{4} v_{6} v_{7}\right\}\right)$ is a 7-vertex twodimensional weak pseudomanifold. From the classification of 7-vertex two-dimensional weak pseudomanifolds, we observe that $\left(\tilde{M}, v_{1} v_{2} v_{3}, v_{4} v_{5} v_{6}, v_{4} v_{6} v_{7}\right)$ is (isomorphic to) $\left(R_{2}, v_{0} v_{1} v_{2}, v_{3} v_{4} v_{5}, v_{3} v_{4} v_{6}\right),\left(R_{3}, v_{0} v_{1} v_{3}, v_{2} v_{4} v_{5}, v_{2} v_{5} v_{6}\right),\left(R_{3}, v_{1} v_{5} v_{6}, v_{0} v_{2} v_{3}, v_{0} v_{2} v_{4}\right)$, $\left(S_{5}, v_{2} v_{3} v_{4}, v_{1} v_{6} v_{7}, v_{1} v_{5} v_{6}\right),\left(S_{6}, v_{1} v_{6} v_{7}, v_{2} v_{3} v_{4}, v_{2} v_{4} v_{5}\right),\left(S_{7}, v_{2} v_{4} v_{5}, v_{3} v_{6} v_{7}, v_{1} v_{3} v_{7}\right)$, $\left(S_{7}, v_{1} v_{3} v_{7}, v_{4} v_{5} v_{6}, v_{2} v_{5} v_{6}\right),\left(S_{7}, v_{1} v_{3} v_{7}, v_{2} v_{4} v_{5}, v_{2} v_{5} v_{6}\right),\left(S_{7}, v_{1} v_{3} v_{7}, v_{2} v_{4} v_{5}, v_{4} v_{5} v_{6}\right)$, $\left(S_{8}, v_{1} v_{2} v_{3}, v_{4} v_{5} v_{6}, v_{5} v_{6} v_{7}\right),\left(S_{8}, v_{2} v_{5} v_{7}, v_{1} v_{3} v_{4}, v_{1} v_{4} v_{6}\right),\left(S_{8}, v_{5} v_{6} v_{7}, v_{1} v_{2} v_{3}, v_{1} v_{3} v_{4}\right)$, $\left(S_{9}, v_{1} v_{2} v_{3}, v_{4} v_{5} v_{6}, v_{5} v_{6} v_{7}\right),\left(Q_{1}, v_{1} v_{2} v_{3}, v_{4} v_{5} v_{7}, v_{4} v_{5} v_{6}\right),\left(Q_{1}, v_{1} v_{2} v_{3}, v_{4} v_{6} v_{7}, v_{4} v_{5} v_{7}\right)$, $\left(Q_{2}, v_{1} v_{2} v_{3}, v_{4} v_{5} v_{7}, v_{5} v_{6} v_{7}\right),\left(P_{1}, v_{1} v_{5} v_{6}, v_{2} v_{3} v_{7}, v_{2} v_{3} v_{4}\right),\left(P_{2}, v_{2} v_{3} v_{7}, v_{1} v_{5} v_{6}, v_{1} v_{4} v_{5}\right)$ or $\left(P_{2}, v_{2} v_{3} v_{4}, v_{1} v_{5} v_{6}, v_{5} v_{6} v_{7}\right)$.

First, assume that $\left(\widetilde{M}, v_{1} v_{2} v_{3}, v_{4} v_{5} v_{6}, v_{4} v_{6} v_{7}\right)$ is $\left(R_{2}, v_{0} v_{1} v_{2}, v_{3} v_{4} v_{5}, v_{3} v_{4} v_{6}\right)$. Let $\widehat{M}$ be the complex obtained from $\widetilde{M}$ by starring a vertex $u_{8}$ in $v_{1} v_{2} v_{3}$ and $u_{9}$ in the edge $v_{4} v_{6}$. Let $\widehat{R_{2}}$ be the complex obtained from $R_{2}$ by starring a vertex $v_{8}$ in $v_{0} v_{1} v_{2}$ and $v_{9}$ in the edge $v_{3} v_{4}$. Then, by Proposition $3.4(\mathrm{a}), \widehat{M} \cong \widehat{R_{2}}$.

The complex obtained from $\widehat{M}$ by identifying $u_{8}$ and $u_{9}$ (to a new vertex $v_{0}$ ) is $M$ and the complex obtained from $\widehat{R_{2}}$ by identifying $v_{8}$ and $v_{9}$ (to a new vertex $v_{7}$ ) is $P_{22}$. Therefore, by Proposition 3.3(b), $M \cong P_{22}$.

Similarly, if $\left(\widetilde{M}, v_{1} v_{2} v_{3}, v_{4} v_{5} v_{6}, v_{4} v_{6} v_{7}\right)$ is one of $\left(R_{3}, v_{0} v_{1} v_{3}, v_{2} v_{4} v_{5}, v_{2} v_{5} v_{6}\right)$, $\left(R_{3}, v_{1} v_{5} v_{6}, v_{0} v_{2} v_{3}, v_{0} v_{2} v_{4}\right),\left(S_{5}, v_{2} v_{3} v_{4}, v_{1} v_{6} v_{7}, v_{1} v_{5} v_{6}\right),\left(S_{6}, v_{1} v_{6} v_{7}, v_{2} v_{3} v_{4}, v_{2} v_{4} v_{5}\right)$, $\left(S_{7}, v_{1} v_{3} v_{7}, v_{4} v_{5} v_{6}, v_{2} v_{5} v_{6}\right),\left(S_{7}, v_{1} v_{3} v_{7}, v_{2} v_{4} v_{5}, v_{2} v_{5} v_{6}\right),\left(S_{7}, v_{1} v_{3} v_{7}, v_{2} v_{4} v_{5}, v_{4} v_{5} v_{6}\right)$, $\left(S_{8}, v_{2} v_{5} v_{7}, v_{1} v_{3} v_{4}, v_{1} v_{4} v_{6}\right),\left(S_{8}, v_{5} v_{6} v_{7}, v_{1} v_{2} v_{3}, v_{1} v_{3} v_{4}\right),\left(Q_{1}, v_{1} v_{2} v_{3}, v_{4} v_{5} v_{7}, v_{4} v_{5} v_{6}\right)$, $\left(Q_{1}, v_{1} v_{2} v_{3}, v_{4} v_{6} v_{7}, v_{4} v_{5} v_{7}\right),\left(P_{1}, v_{1} v_{5} v_{6}, v_{2} v_{3} v_{7}, v_{2} v_{3} v_{4}\right)$ or $\left(P_{2}, v_{2} v_{3} v_{4}, v_{1} v_{5} v_{6}\right.$, $\left.v_{5} v_{6} v_{7}\right)$, then $M$ is $P_{23}, P_{25}, P_{6}, P_{11}, P_{4}, P_{9}, P_{3}, P_{15}, P_{13}, Q_{7}, Q_{8}, P_{34}$ or $P_{28}$ respectively.

If $\left(\tilde{M}, v_{1} v_{2} v_{3}, v_{4} v_{5} v_{6}, v_{4} v_{6} v_{7}\right)$ is $\left(S_{7}, v_{2} v_{4} v_{5}, v_{3} v_{6} v_{7}, v_{1} v_{3} v_{7}\right),\left(S_{8}, v_{1} v_{2} v_{3}, v_{4} v_{5} v_{6}\right.$, $\left.v_{5} v_{6} v_{7}\right),\left(S_{9}, v_{1} v_{2} v_{3}, v_{4} v_{5} v_{6}, v_{5} v_{6} v_{7}\right),\left(Q_{2}, v_{1} v_{2} v_{3}, v_{4} v_{5} v_{7}, v_{5} v_{6} v_{7}\right)$ or $\left(P_{2}, v_{2} v_{3} v_{7}\right.$, $v_{1} v_{5} v_{6}, v_{1} v_{4} v_{5}$ ) then $M$ is isomorphic to $P_{6}, P_{11}, P_{15}, Q_{11}$ or $P_{30}$ respectively.

Lemma 5.4. If $M$ has no singular vertex of degree 7 , then $M$ is isomorphic to $P_{5}, P_{7}, P_{8}$, $P_{10}, P_{12}, P_{14}, P_{16}, \ldots, P_{21}, P_{24}, P_{26}, P_{27}, P_{33}, P_{36}, P_{39}, Q_{4}, Q_{9}, Q_{10}, Q_{13}$ or $Q_{15}$.

Proof. Since $M$ is not a combinatorial 2-manifold, there exists a vertex, say $v_{0}$, whose link is of the form $C_{3}\left(v_{1}, v_{2}, v_{3}\right) \sqcup C_{3}\left(v_{4}, v_{5}, v_{6}\right)$. Since $M ¥ Q_{1}$, at most one of $v_{1} v_{2} v_{3}$ or $v_{4} v_{5} v_{6}$ can be a simplex. Let $v_{7}$ be the remaining vertex of $M$.

Case I. Exactly one of $v_{1} v_{2} v_{3}$ or $v_{4} v_{5} v_{6}$ is a simplex. Assume, without loss of generality, that $v_{1} v_{2} v_{3}$ is a simplex. Then the triangle $\left(\neq v_{0} v_{4} v_{5}\right)$ through $v_{4} v_{5}$ is $v_{1} v_{4} v_{5}, v_{2} v_{4} v_{5}$, $v_{3} v_{4} v_{5}$ or $v_{4} v_{5} v_{7}$. Without loss of generality, we can assume that either $v_{1} v_{4} v_{5}$ or $v_{4} v_{5} v_{7}$ is a simplex.

In the first case, $M$ is isomorphic to $Q_{9}, Q_{13}$ or $Q_{15}$.

In the second case, $M$ is isomorphic to $Q_{4}, Q_{9}$ or $Q_{13}$.

Case II. Neither $v_{1} v_{2} v_{3}$ nor $v_{4} v_{5} v_{6}$ is a simplex. Then $\tilde{M}=\left(M \backslash\left\{v_{0}\right\}\right) \cup\left\{v_{1} v_{2} v_{3}, v_{4} v_{5} v_{6}\right\}$ is a 7 -vertex two-dimensional weak pseudomanifold. Since $M$ has no singular vertex of degree 
7, none of $v_{1}, \ldots, v_{6}$ is singular in $\tilde{M}$. From the classification of 7-vertex two-dimensional weak pseudomanifolds we observe that $\left(\tilde{M}, v_{1} v_{2} v_{3}, v_{4} v_{5} v_{6}\right)$ is (isomorphic to) $\left(T_{1}, v_{1} v_{2} v_{4}, v_{3} v_{5} v_{6}\right),\left(R_{2}, v_{0} v_{1} v_{3}, v_{2} v_{5} v_{6}\right),\left(R_{3}, v_{0} v_{1} v_{3}, v_{2} v_{4} v_{5}\right),\left(R_{3}, v_{0} v_{1} v_{3}, v_{2} v_{5} v_{6}\right)$, $\left(R_{4}, v_{0} v_{1} v_{3}, v_{2} v_{4} v_{5}\right),\left(S_{5}, v_{2} v_{3} v_{4}, v_{1} v_{6} v_{7}\right),\left(S_{5}, v_{2} v_{3} v_{4}, v_{1} v_{5} v_{6}\right),\left(S_{6}, v_{2} v_{3} v_{4}, v_{1} v_{6} v_{7}\right)$, $\left(S_{7}, v_{1} v_{2} v_{3}, v_{4} v_{5} v_{6}\right),\left(S_{7}, v_{1} v_{2} v_{7}, v_{3} v_{4} v_{6}\right),\left(S_{7}, v_{1} v_{2} v_{7}, v_{4} v_{5} v_{6}\right),\left(S_{7}, v_{1} v_{3} v_{7}, v_{4} v_{5} v_{6}\right)$, $\left(S_{8}, v_{1} v_{2} v_{3}, v_{4} v_{5} v_{6}\right),\left(S_{8}, v_{1} v_{3} v_{4}, v_{5} v_{6} v_{7}\right),\left(S_{8}, v_{1} v_{2} v_{7}, v_{4} v_{5} v_{6}\right),\left(S_{9}, v_{1} v_{2} v_{3}, v_{4} v_{5} v_{6}\right)$, $\left(Q_{1}, v_{1} v_{2} v_{3}, v_{4} v_{5} v_{6}\right),\left(P_{1}, v_{1} v_{2} v_{6}, v_{3} v_{4} v_{5}\right)$ or $\left(P_{2}, v_{1} v_{5} v_{6}, v_{2} v_{3} v_{4}\right)$.

If $\left(\widetilde{M}, v_{1} v_{2} v_{3}, v_{4} v_{5} v_{6}\right)$ is $\left(T_{1}, v_{1} v_{2} v_{4}, v_{3} v_{5} v_{6}\right)$ then, by Proposition $3.5(\mathrm{~b}), M$ is $P_{39}$.

Similarly, if $\left(\tilde{M}, v_{1} v_{2} v_{3}, v_{4} v_{5} v_{6}\right)$ is $\left(R_{2}, v_{0} v_{1} v_{3}, v_{2} v_{5} v_{6}\right), \ldots,\left(S_{9}, v_{1} v_{2} v_{3}, v_{4} v_{5} v_{6}\right)$, $\left(Q_{1}, v_{1} v_{2} v_{3}, v_{4} v_{5} v_{6}\right),\left(P_{1}, v_{1} v_{2} v_{6}, v_{3} v_{4} v_{5}\right)$ or $\left(P_{2}, v_{1} v_{5} v_{6}, v_{2} v_{3} v_{4}\right)$, then $M$ is $P_{24}, P_{26}$, $P_{27}, P_{21}, P_{7}, P_{5}, P_{8}, P_{12}, P_{10}, P_{14}, P_{17}, P_{18}, P_{19}, P_{16}, P_{20}, Q_{10}, P_{36}$ or $P_{33}$ respectively. $\square$

Lemma 5.5. The weak pseudomanifolds mentioned in Theorem 1.2 are pairwise nonisomorphic.

Proof. First, we observe that all the $P_{i}$ 's $(1 \leq i \leq 39)$ are pseudomanifolds, while all the $Q_{i}$ 's $(1 \leq i \leq 16)$ are not pseudomanifolds.

For $3 \leq i<j \leq 39, P_{i} \cong P_{j}$ implies that the degree sequences of $P_{i}$ and $P_{j}$ are the same and the singular degree sequences of $P_{i}$ and $P_{j}$ are the same and hence, from the description of $P_{i}$ 's in $\S 2,(i, j) \in\{(17,18),(19,20),(22,25)\}$.

If there exists an isomorphism $\alpha: P_{17} \rightarrow P_{18}$, then $\alpha\left(v_{0}\right)=v_{0}$ (since, these are the only singular vertices). In $P_{17}$, each of the $C_{3}$ 's in the link of $v_{0}$ has a vertex of degree 4 , while only one $C_{3}$ in $\operatorname{Lk}\left(v_{0}\right)$ in $P_{18}$ has a vertex of degree 4 . Therefore, $\alpha$ is not an isomorphism.

If $\beta: P_{19} \rightarrow P_{20}$ is an isomorphism, then $\beta\left(v_{0}\right)=v_{0}$ (since, these are the only singular vertices). We see that $\operatorname{Lk}\left(v_{0}\right)$ in $P_{19}$ has a vertex (namely, $\left.v_{3}\right)$ of degree 4 whereas $\operatorname{Lk}\left(v_{0}\right)$ in $P_{20}$ has no vertex of degree 4 . So, $P_{19} \not P_{20}$.

If there exists an isomorphism $\psi: P_{22} \rightarrow P_{25}$, then $\psi\left(v_{7}\right)=v_{7}$ (since, these are the only singular vertices of degree 7). In $P_{25}$ the link of $v_{7}$ has a $C_{3}$ all of whose vertices have degree 6 but the link of $v_{7}$ in $P_{22}$ has no such $C_{3}$. So, $P_{22} \not P_{25}$.

For $3 \leq i<j \leq 16, Q_{i} \cong Q_{j}$ implies that the degree sequences of $Q_{i}$ and $Q_{j}$ are the same and hence, from the description of $Q_{i}$ 's in $\S 2,(i, j)=(6,7)$.

Two degree 3 vertices in $Q_{6}$ form an edge but that is not the case in $Q_{7}$. Thus, $Q_{6} ¥ Q_{7}$. This completes the proof of the lemma.

Proof of Theorem 1.2. Let $M$ be a two-dimensional 8-vertex weak pseudomanifold which is not a combinatorial 2-manifold. Then $M$ has a singular vertex.

First consider the case when $M$ has a singular vertex, say $v_{0}$, of degree 7 . Then the link of $v_{0}$ is of the form $C_{3} \sqcup C_{4}$. Let $\operatorname{Lk}\left(v_{0}\right)=C_{3}\left(v_{1}, v_{2}, v_{3}\right) \sqcup C_{4}\left(v_{4}, v_{5}, v_{6}, v_{7}\right)$.

If $v_{1} v_{2} v_{3} \in M$ then, by Lemma 5.1, $M$ is isomorphic to $Q_{3}, Q_{5}, Q_{6}, Q_{12}$ or $Q_{16}$.

If $v_{1} v_{2} v_{3}$ is not a simplex then we have two cases, namely, (i) either both $v_{4} v_{6}$ and $v_{5} v_{7}$ are edges of $M$ or (ii) at least one of $v_{4} v_{6}$ or $v_{5} v_{7}$ is a non-edge of $M$, say (without loss of generality) $v_{4} v_{6}$ is not an edge. Then, by Lemmas 5.2 and 5.3, $M$ is isomorphic to $P_{3}, P_{4}$, $P_{6}, P_{9}, P_{11}, P_{13}, P_{15}, P_{22}, P_{23}, P_{25}, P_{28}, \ldots, P_{32}, P_{34}, P_{35}, P_{37}, P_{38}, Q_{7}, Q_{8}, Q_{11}$ or $Q_{14}$.

Finally, consider the case when $M$ has no singular vertex of degree 7 . In this case, by Lemma 5.4, $M$ is isomorphic to $P_{5}, P_{7}, P_{8}, P_{10}, P_{12}, P_{14}, P_{16}, \ldots, P_{21}, P_{24}, P_{26}, P_{27}$, $P_{33}, P_{36}, P_{39}, Q_{4}, Q_{9}, Q_{10}, Q_{13}$ or $Q_{15}$. This completes the proof. 


\section{Applications}

Proof of Corollary 1.3. Observe that $S_{1}, \ldots, S_{23}$ triangulate $S^{2}, R_{1}, \ldots, R_{20}$ triangulate $\mathbb{R} P^{2}, T_{1}, \ldots, T_{8}$ triangulate $S^{1} \times S^{1}, K_{1}, \ldots, K_{6}$ triangulate the Klein bottle $(K)$ and $D$ triangulates $S^{2} \sqcup S^{2}$. These, Proposition 3.1 and Theorem 1.1 imply Corollary 1.3(i).

By Proposition 3.3(c), $P_{1}, \ldots, P_{20}$ triangulate the pinched sphere $(P), P_{21}, \ldots, P_{27}$ triangulate $\mathbb{R} P^{2} \# P, P_{28}, \ldots, P_{36}$ triangulate $P \# P, P_{37}$ triangulates $\mathbb{R} P^{2} \# P \# P, P_{38}$ triangulates $K \# P$ and $P_{39}$ triangulates $\left(S^{1} \times S^{1}\right) \# P$.

Also (from the pictures in $\S 2$ ) $Q_{1}, Q_{3}$ and $Q_{4}$ triangulate the union of two $S^{2}$ 's having one point in common, $Q_{2}, Q_{5}, \ldots, Q_{10}$ triangulate the union of two $S^{2}$ 's having two points in common, $Q_{11}, Q_{12}$ and $Q_{13}$ triangulate the union of two 2-spheres having three points in common, $Q_{14}$ and $Q_{15}$ triangulate the union of two 2-spheres having four points in common, $Q_{16}$ triangulates the union of $S^{2}$ and $\mathbb{R} P^{2}$ having three points in common. These, Proposition 3.1 and Theorem 1.2 imply Corollary 1.3(ii).

Proof of Theorem 1.4. Let $\varphi: K_{n}^{2} \rightarrow S_{4}^{2}$ be a simplicial map, where $K_{n}^{2}$ is an oriented $n$-vertex two-dimensional pseudomanifold and $S_{4}^{2}$ is the 4-vertex 2-sphere with an orientation.

If $n \leq 7$, then there exists a vertex, say $a$, of $S_{4}^{2}$ whose inverse image contains less than 2 vertices and hence there exists a triangle through $a$ whose inverse image contains less than 3 triangles. Thus, $\operatorname{deg}(\varphi)<3$.

If $n=8$, then $f_{2}\left(K_{8}^{2}\right) \leq 18$ and hence $\operatorname{deg}(\varphi) \leq 18 / 4$. Let $\varphi$ be of type $\left(n_{1}, n_{2}, n_{3}, n_{4}\right)$. Assume, $\operatorname{deg}(\varphi) \geq 3$. By the same argument as above, each vertex of $S_{4}^{2}$ has two inverse images. Let $\varphi^{-1}(a)=\left\{a_{1}, a_{2}\right\}, \varphi^{-1}(b)=\left\{b_{1}, b_{2}\right\}, \varphi^{-1}(c)=\left\{c_{1}, c_{2}\right\}$ and $\varphi^{-1}(d)=$ $\left\{d_{1}, d_{2}\right\}$, where $a, b, c$ and $d$ are the vertices of $S_{4}^{2}$. So, there does not exist any 2-simplex $\sigma$ such that $\varphi(\sigma)$ is a vertex and hence we have:

Claim 6.1. Each collapsing triangle contains exactly one collapsing edge. On the other hand, both the triangles through a collapsing edge are collapsing.

It is also easy to see the following:

Claim 6.2. If $S_{a}:=\left\{\sigma \in K_{8}^{2}: \varphi(\sigma)\right.$ is a 2 -simplex containing $\left.a\right\}$, then $\#\left(S_{a}\right) \leq 12$. Further, if $a_{1} a_{2}$ is an edge then \# $\left(S_{a}\right) \leq\left(\operatorname{deg}\left(a_{1}\right)-2\right)+\left(\operatorname{deg}\left(a_{2}\right)-2\right) \leq 10$.

If $\operatorname{deg}(\varphi)=4$, then $f_{2}\left(K_{8}^{2}\right) \geq 16$ and hence $\chi\left(K_{8}^{2}\right) \leq 0$. If $\chi\left(K_{8}^{2}\right)=-1$ then by Theorems 1.1 and 1.2, $K_{8}^{2}=P_{37}, P_{38}$ or $P_{39}$. Since $P_{37}$ and $P_{38}$ are non-orientable, $K_{8}^{2}=P_{39}$. Clearly, from the degree sequence of $P_{39}$, there exists an edge $x y$ such that $\varphi(x)=\varphi(y)$. Then (by Claim 6.2) $\operatorname{deg}(\varphi) \leq 10 / 3$, a contradiction. So, $\chi\left(K_{8}^{2}\right)=0$ and hence $f_{2}\left(K_{8}^{2}\right)=16$. Then $\varphi$ is of type $(4,4,4,4)$ and hence there is no collapsing 2simplex and hence, by Claim 6.1, no collapsing edge. So, if $\varphi(u)=\varphi(v)$, then $u v$ is a non-edge and conversely (since the number of non-edges is $\left(\begin{array}{l}8 \\ 2\end{array}\right)-24=4$ ). These imply that the 4 non-edges are disjoint and hence the degree sequence of $K_{8}^{2}$ is $6^{8}$. Then, by Theorems 1.1 and 1.2, $K_{8}^{2}=T_{2}$ or $P_{36}$. In both the cases $v_{3} v_{6}$ is a non-edge and hence $\varphi\left(v_{3}\right)=\varphi\left(v_{6}\right)$. If $K_{8}^{2}=T_{2}$ then $\varphi\left(v_{2} v_{3} v_{7}\right)$ and $\varphi\left(v_{2} v_{6} v_{7}\right)$ are the same 2-simplex, a contradiction to Proposition 3.6(b). If $K_{8}^{2}=P_{36}$ then $\varphi\left(v_{2} v_{3} v_{4}\right)$ and $\varphi\left(v_{2} v_{4} v_{6}\right)$ are the same 2-simplex, a contradiction to Proposition 3.6(b). Thus $\operatorname{deg}(\varphi) \leq 3$. This proves the first part of the theorem.

Now assume $\operatorname{deg}(\varphi)=3$. In this case \#(Sa) $\geq 9$ for each vertex $a$ of $S_{4}^{2}$. Thus, $f_{2}\left(K_{8}^{2}\right) \geq 12$ and hence, by Theorems 1.1 and $1.2,-1 \leq \chi\left(K_{8}^{2}\right) \leq 2$. 
Case I. $\chi\left(K_{8}^{2}\right)=-1$. By Theorem $1.2\left(\right.$ as $K_{8}^{2}$ is orientable), $K_{8}^{2}=P_{39}$. Let $\varphi\left(v_{0}\right)=a$. If $\varphi\left(v_{7}\right)=a$ then (since \# $\left.\left(S_{a}\right) \geq 9\right) \varphi\left(v_{1}\right), \varphi\left(v_{2}\right), \varphi\left(v_{4}\right)$ are distinct and $\varphi\left(v_{3}\right), \varphi\left(v_{5}\right)$, $\varphi\left(v_{6}\right)$ are distinct. Again, $\varphi\left(v_{1}\right)=\varphi\left(v_{3}\right)$ or $\varphi\left(v_{5}\right)$ implies that there are 4 collapsing 2 -simplices through $v_{7}$. This implies, \# $\left(S_{a}\right) \leq 8$, a contradiction. So, $\varphi\left(v_{1}\right)=\varphi\left(v_{6}\right)$. Similarly, $\varphi\left(v_{2}\right)=\varphi\left(v_{5}\right)$ and $\varphi\left(v_{3}\right)=\varphi\left(v_{4}\right)$. Then $\varphi^{-1}(b c d)$ contains no 2-simplices, a contradiction. So, $v_{7} \notin \varphi^{-1}(a)$. If $v_{1} \in \varphi^{-1}(a)$ then $\varphi\left(v_{0} v_{5} v_{6}\right)=\varphi\left(v_{1} v_{5} v_{6}\right)$ and hence, by Proposition 3.6(c), \#( $\left.S_{a}\right) \geq 9+2=11$. On the other hand \# $\left(S_{a}\right) \leq\left(\operatorname{deg}\left(v_{0}\right)-2\right)+$ $\left(\operatorname{deg}\left(v_{1}\right)-2\right)=9$, a contradiction. Similarly, for $v_{2}, v_{3}, v_{4}, v_{5}$ or $v_{6} \in \varphi^{-1}(a)$ we get contradictions.

Case II. $\chi\left(K_{8}^{2}\right)=0$. By Claim 6.1, the inverse image of each triangle of $S_{4}^{2}$ contains 3 , 5 or 7 triangles. Also, by Claim $6.2,\left(n_{1}, n_{2}, n_{3}, n_{4}\right)=(7,3,3,3)$ or $(5,5,3,3)$ is not possible. So, $\left(n_{1}, n_{2}, n_{3}, n_{4}\right)$ is $(5,3,3,3)$ or $(3,3,3,3)$.

Subcase II.1. If $\left(n_{1}, n_{2}, n_{3}, n_{4}\right)=(5,3,3,3)$, then we have two collapsing triangles and hence, by Claim 6.1, exactly one collapsing edge. Assume (if necessary, by taking a composition with an automorphism of $\left.S_{4}^{2}\right)$ that the number of triangles of $\varphi^{-1}(a b c)$ is 5 . Then $\#\left(S_{a}\right) \geq 5+3+3=11$ and hence, by Claim 6.2, $a_{1} a_{2}$ is not an edge. So, $11 \leq$ $\operatorname{deg}\left(a_{1}\right)+\operatorname{deg}\left(a_{2}\right) \leq 12$. Similarly, $b_{1} b_{2}$ and $c_{1} c_{2}$ are not edges, $11 \leq \operatorname{deg}\left(b_{1}\right)+\operatorname{deg}\left(b_{2}\right)$, $\operatorname{deg}\left(c_{1}\right)+\operatorname{deg}\left(c_{2}\right) \leq 12$ and $\#\left(S_{b}\right), \#\left(S_{c}\right) \geq 11$. So, $d_{1} d_{2}$ is the collapsing edge. Without loss of generality, let $\operatorname{deg}\left(a_{1}\right)=\operatorname{deg}\left(b_{1}\right)=\operatorname{deg}\left(c_{1}\right)=6$.

Since the sum of the degrees of all the vertices is 48 , we get $12 \leq \operatorname{deg}\left(d_{1}\right)+\operatorname{deg}\left(d_{2}\right) \leq 14$. If $\operatorname{deg}\left(d_{1}\right)+\operatorname{deg}\left(d_{2}\right)=12$ then (by Claim 6.2) \#( $\left.S_{d}\right) \leq 12-4$, a contradiction. If $\operatorname{deg}\left(d_{1}\right)+\operatorname{deg}\left(d_{2}\right)=14$ then, $\operatorname{deg}\left(d_{1}\right)=\operatorname{deg}\left(d_{2}\right)=7$. Without loss, we can assume that $\operatorname{deg}\left(a_{2}\right)=\operatorname{deg}\left(b_{2}\right)=5$ and $\operatorname{deg}\left(c_{2}\right)=6$. Let $x, y$ be the vertices of $K_{8}^{2}$ such that $d_{1} d_{2} x$ and $d_{1} d_{2} y$ are 2 -simplices. If $x=a_{1}$, then $\#\left(S_{a}\right) \leq 6+5-1=10$, a contradiction. By a similar argument we see that $x, y \notin\left\{a_{1}, a_{2}, b_{1}, b_{2}\right\}$. Hence $x, y \in\left\{c_{1}, c_{2}\right\}$. Then $\#\left(S_{c}\right) \leq 12-2=10$, a contradiction. Thus, $\operatorname{deg}\left(d_{1}\right)+\operatorname{deg}\left(d_{2}\right)=13$.

We can assume (if necessary, by taking composition with automorphisms of $S_{4}^{2}$ and $K_{8}^{2}$, i.e., up to an equivalence) that $\operatorname{deg}\left(d_{1}\right)=7, \operatorname{deg}\left(d_{2}\right)=6, \operatorname{deg}\left(b_{2}\right)=\operatorname{deg}\left(c_{2}\right)=6$ and $\operatorname{deg}\left(a_{2}\right)=5$. Hence there is no collapsing 2-simplex through $a_{1}$ or $a_{2}$. So, the degree sequence of $K_{8}^{2}$ is $7 \cdot 6^{6} \cdot 5$ and hence, by Theorems 1.1 and $1.2, K_{8}^{2}=P_{35}$ and $d_{1}=v_{0}$, $a_{2}=v_{1}$. We can also assume that $\left\{b_{1}, b_{2}\right\}=\left\{v_{2}, v_{6}\right\}$ and $\left\{c_{1}, c_{2}\right\}=\left\{v_{3}, v_{6}\right\}$. Then, $\left\{a_{1}, d_{2}\right\}=\left\{v_{5}, v_{7}\right\}$.

If $\left(a_{1}, d_{2}\right)=\left(v_{7}, v_{5}\right)$ then $\left\{v_{1} v_{2} v_{4}, v_{7} v_{2} v_{4}, v_{1} v_{3} v_{6}, v_{7} v_{3} v_{6}\right\} \subseteq S_{a}$. This implies, by Proposition 3.6(c), \# $\left(S_{a}\right) \geq 13$, a contradiction. So, $\left(a_{1}, d_{2}\right)=\left(v_{5}, v_{7}\right)$. Then, $\varphi$ is equivalent to $h$.

Subcase II.2. If $\left(n_{1}, n_{2}, n_{3}, n_{4}\right)=(3,3,3,3)$, then we have 4 collapsing triangles and hence, by Claim 6.1, exactly 2 collapsing edges.

Without loss of generality, we can assume that $a_{1} a_{2}$ and $b_{1} b_{2}$ are edges whereas $c_{1} c_{2}$ and $d_{1} d_{2}$ are not. Then $9 \leq \operatorname{deg}\left(c_{1}\right)+\operatorname{deg}\left(c_{2}\right), \operatorname{deg}\left(d_{1}\right)+\operatorname{deg}\left(d_{2}\right) \leq 12$. We see that $\left(\operatorname{deg}\left(a_{1}\right)-2\right)+\left(\operatorname{deg}\left(a_{2}\right)-2\right)=\#\left(S_{a}\right)=9$. Hence $\operatorname{deg}\left(a_{1}\right)+\operatorname{deg}\left(a_{2}\right)=13$. Similarly, $\operatorname{deg}\left(b_{1}\right)+\operatorname{deg}\left(b_{2}\right)=13$. We can assume that $\operatorname{deg}\left(a_{1}\right)=\operatorname{deg}\left(b_{1}\right)=7$ and $\operatorname{deg}\left(a_{2}\right)=$ $\operatorname{deg}\left(b_{2}\right)=6$. Thus, $\operatorname{deg}\left(c_{1}\right)+\operatorname{deg}\left(c_{2}\right)+\operatorname{deg}\left(d_{1}\right)+\operatorname{deg}\left(d_{2}\right)=22$. This shows that $10 \leq \operatorname{deg}\left(c_{1}\right)+\operatorname{deg}\left(c_{2}\right), \operatorname{deg}\left(d_{1}\right)+\operatorname{deg}\left(d_{2}\right) \leq 12$. Since $\operatorname{deg}\left(c_{1}\right), \operatorname{deg}\left(c_{2}\right) \leq 6$, it is clear that $\operatorname{deg}\left(c_{1}\right), \operatorname{deg}\left(c_{2}\right) \geq 4$. Thus, there exists no vertex of degree 3 . We may assume that $\operatorname{deg}\left(c_{1}\right)+\operatorname{deg}\left(c_{2}\right) \geq \operatorname{deg}\left(d_{1}\right)+\operatorname{deg}\left(d_{2}\right), \operatorname{deg}\left(c_{1}\right) \geq \operatorname{deg}\left(c_{2}\right)$ and $\operatorname{deg}\left(d_{1}\right) \geq \operatorname{deg}\left(d_{2}\right)$. Then we have the following three possibilities for the degrees of the remaining four vertices. 
(II.2.1) $\operatorname{deg}\left(c_{1}\right)=\operatorname{deg}\left(c_{2}\right)=\operatorname{deg}\left(d_{1}\right)=6$ and $\operatorname{deg}\left(d_{2}\right)=4$.

(II.2.2) $\operatorname{deg}\left(c_{1}\right)=\operatorname{deg}\left(c_{2}\right)=6$ and $\operatorname{deg}\left(d_{1}\right)=\operatorname{deg}\left(d_{2}\right)=5$.

(II.2.3) $\operatorname{deg}\left(c_{1}\right)=\operatorname{deg}\left(d_{1}\right)=6$ and $\operatorname{deg}\left(c_{2}\right)=\operatorname{deg}\left(d_{2}\right)=5$.

(II.2.1) In this case, from Theorems 1.1 and 1.2, we see that $K_{8}^{2}$ is $T_{4}$. The vertex of degree 4 of $T_{4}$ is $v_{0}$. Hence $d_{2}=v_{0}$. We can assume, without loss of generality, that $a_{1}=v_{7}$ and $b_{1}=v_{2}$. Since $v_{4}$ and $v_{5}$ are the only vertices of degree 6 which do not form an edge, we can assume $c_{1}=v_{4}$ and $c_{2}=v_{5}$. Then $\varphi^{-1}(a c d)$ contains $v_{0} v_{4} v_{7}$ and $v_{0} v_{5} v_{7}$, a contradiction to Proposition 3.6(b) (since, \# $\left(\varphi^{-1}(\sigma)\right)=3=\operatorname{deg}(\varphi)$, for each 2-simplex $\sigma$ in $S_{4}^{2}$ ).

(II.2.2) In this case, the degree sequence of $K_{8}^{2}$ is $7^{2} \cdot 6^{4} \cdot 5^{2}$ and hence, by Theorems 1.1 and $1.2, K_{8}^{2}$ is $T_{5}, T_{8}, K_{1}, P_{26}, P_{32}, P_{33}$ or $P_{34}$. As $K_{1}$ and $P_{26}$ are non-orientable, $K_{8}^{2}$ is not $K_{1}$ or $P_{26}$. Since the two degree 5 vertices of $T_{5}$ form an edge, $K_{8}^{2} \neq T_{5}$.

If $K_{8}^{2}$ is $T_{8}$ then $\left\{d_{1}, d_{2}\right\}=\left\{v_{0}, v_{3}\right\}$ and $\left\{a_{1}, b_{1}\right\}=\left\{v_{2}, v_{5}\right\}$. Then $\varphi^{-1}(a b d)$ contains $v_{0} v_{2} v_{5}$ and $v_{2} v_{3} v_{5}$, a contradiction to Proposition 3.6(b).

If $K_{8}^{2}=P_{32}$ then $\left\{d_{1}, d_{2}\right\}=\left\{v_{3}, v_{6}\right\}$ and we can assume $a_{1}=v_{0}, b_{1}=v_{7}$. Then $\left\{c_{1}, c_{2}\right\}=\left\{v_{1}, v_{5}\right\}$ and $\left\{a_{2}, b_{2}\right\}=\left\{v_{2}, v_{4}\right\}$. If $\left(a_{2}, b_{2}\right)=\left(v_{2}, v_{4}\right)$ then $v_{0} v_{1} v_{2}, v_{0} v_{2} v_{3}$, $v_{0} v_{4} v_{7}, v_{2} v_{4} v_{7}$ are collapsing and hence $\#\left(S_{a}\right) \leq 7$, a contradiction. If $\left(a_{2}, b_{2}\right)=\left(v_{4}, v_{2}\right)$ then $v_{0} v_{4} v_{5}, v_{0} v_{4} v_{7}, v_{2} v_{4} v_{7}, v_{2} v_{5} v_{7}$ are collapsing and hence $\#\left(S_{a}\right) \leq 8$, a contradiction.

If $K_{8}^{2}=P_{33}$ then $\left\{d_{1}, d_{2}\right\}=\left\{v_{3}, v_{5}\right\}$ and $\left\{a_{1}, b_{1}\right\}=\left\{v_{1}, v_{4}\right\}$. Then $\varphi^{-1}(a b d)$ contains $v_{1} v_{3} v_{4}$ and $v_{1} v_{4} v_{5}$, a contradiction to Proposition 3.6(b).

If $K_{8}^{2}=P_{34}$ then $\left\{d_{1}, d_{2}\right\}=\left\{v_{2}, v_{3}\right\}$ and $\left\{a_{1}, b_{1}\right\}=\left\{v_{0}, v_{7}\right\}$. Then $\varphi^{-1}(a b d)$ contains $v_{0} v_{2} v_{7}$ and $v_{0} v_{3} v_{7}$, a contradiction to Proposition 3.6(b).

(II.2.3) In this case also, the degree sequence of $K_{8}^{2}$ is $7^{2} \cdot 6^{4} \cdot 5^{2}$ and hence (since $K_{8}^{2}$ is orientable), by Theorems 1.1 and $1.2, K_{8}^{2}$ is $T_{5}, T_{8}, P_{32}, P_{33}$ or $P_{34}$.

If $K_{8}^{2}=T_{5}$ then assume (up to an equivalence) that, $a_{1}=v_{1}, b_{1}=v_{2}, c_{2}=v_{0}$, $d_{2}=v_{5}$. Then, $v_{4}=d_{1}$. These imply, $\varphi\left(v_{0} v_{2} v_{4}\right)=\varphi\left(v_{0} v_{2} v_{5}\right)=b c d$, a contradiction to Proposition 3.6(b).

If $K_{8}^{2}=T_{8}$ then assume, without loss of generality, that $a_{1}=v_{2}, b_{1}=v_{5}, c_{2}=v_{0}$ and $d_{2}=v_{3}$. Then $c_{1}=v_{1}$ and $d_{1}=v_{6}$. So, $\left\{a_{2}, b_{2}\right\}=\left\{v_{4}, v_{7}\right\}$. If $\left(a_{2}, b_{2}\right)=\left(v_{4}, v_{7}\right)$ the $\varphi\left(v_{2} v_{3} v_{7}\right)=\varphi\left(v_{2} v_{6} v_{7}\right)=a b d$, a contradiction to Proposition 3.6(b). If $\left(a_{2}, b_{2}\right)=$ $\left(v_{7}, v_{4}\right)$ the $\varphi\left(v_{0} v_{5} v_{7}\right)=\varphi\left(v_{1} v_{5} v_{7}\right)=a b c$, a contradiction to Proposition 3.6(b).

If $K_{8}^{2}=P_{32}$ then we can assume (up to an equivalence) $a_{1}=v_{0}, b_{1}=v_{7}, c_{2}=v_{3}$, $d_{2}=v_{6}$ and hence $c_{1}=v_{4}$ and $d_{1}=v_{2}$. Then $\varphi\left(v_{1} v_{2} v_{4}\right)=\varphi\left(v_{1} v_{4} v_{6}\right)$, a contradiction to Proposition 3.6(b).

If $K_{8}^{2}=P_{33}$ then assume, without loss of generality, that $a_{1}=v_{1}, b_{1}=v_{4}, c_{2}=v_{3}$ and $d_{2}=v_{5}$. Then $c_{1}=v_{6}$ and $d_{1}=v_{2}$. Clearly, $\left(a_{2}, b_{2}\right)=\left(v_{0}, v_{7}\right)$ or $\left(v_{7}, v_{0}\right)$. In the first case $\varphi\left(v_{1} v_{3} v_{4}\right)=\varphi\left(v_{0} v_{3} v_{4}\right)=a b c$, a contradiction to Proposition 3.6(b). In the second case, $\varphi\left(v_{1} v_{4} v_{5}\right)=\varphi\left(v_{4} v_{5} v_{7}\right)=a b d$, a contradiction to Proposition 3.6(b) again.

If $K_{8}^{2}=P_{34}$ then assume (up to an equivalence) that, $a_{1}=v_{0}, b_{1}=v_{7}, c_{2}=v_{2}$ and $d_{2}=v_{3}$. Then $c_{1}=v_{5}, d_{1}=v_{6}$ and $\left\{a_{2}, b_{2}\right\}=\left\{v_{1}, v_{4}\right\}$. If $\left(a_{2}, b_{2}\right)=\left(v_{1}, v_{4}\right)$ then $\varphi\left(v_{0} v_{3} v_{7}\right)=\varphi\left(v_{1} v_{3} v_{7}\right)=a b d$, a contradiction to Proposition 3.6(b). So, $\left(a_{2}, b_{2}\right)=$ $\left(v_{4}, v_{1}\right)$. In this case, $\varphi$ is equivalent to $g$.

Case III. $\chi\left(K_{8}^{2}\right)=2$. In this case $f_{2}\left(K_{8}^{2}\right)=12$ and hence there is no collapsing 2simplex and $\left(n_{1}, n_{2}, n_{3}, n_{4}\right)=(3,3,3,3)$. So, $\operatorname{deg}\left(a_{1}\right)+\operatorname{deg}\left(a_{2}\right)=\operatorname{deg}\left(b_{1}\right)+\operatorname{deg}\left(b_{2}\right)=$ $\operatorname{deg}\left(c_{1}\right)+\operatorname{deg}\left(c_{2}\right)=\operatorname{deg}\left(d_{1}\right)+\operatorname{deg}\left(d_{2}\right)=9$. Further, by Claim 6.1, $a_{1} a_{2}, b_{1} b_{2}, c_{1} c_{2}$ and $d_{1} d_{2}$ are not edges. 
Observe that $K_{8}^{2}$ cannot have a vertex of degree 4. (If possible let there exist a vertex, say $a_{1}$, of degree 4 . Let $b_{1} c_{1}, c_{1} d_{1}, d_{1} x$ and $x b_{1}$ be the edges in $\operatorname{Lk}\left(a_{1}\right)$. Clearly, $x \neq a_{2}$. If $x=b_{2}$ or $d_{2}$ then there is a collapsing 2-simplex, a contradiction. If $x=c_{2}$ then Proposition 3.6(b) is contradicted.) Hence, by Theorems 1.1 and $1.2, K_{8}^{2}$ is $S_{15}$ or $S_{20}$. But, $S_{20}$ has no pair of vertices, the sum of whose degrees is 9 . So, $K_{8}^{2}$ is $S_{15}$.

If $\operatorname{deg}\left(a_{1}\right) \geq \operatorname{deg}\left(a_{2}\right)$, then $\left(a_{1}, a_{2}\right) \in\left\{\left(v_{1}, v_{5}\right),\left(v_{2}, v_{0}\right),\left(v_{4}, v_{7}\right),\left(v_{6}, v_{3}\right)\right\}$. We observe that $\left(v_{1}, v_{2}\right)\left(v_{5}, v_{0}\right),\left(v_{1}, v_{4}\right)\left(v_{5}, v_{7}\right),\left(v_{1}, v_{6}\right)\left(v_{3}, v_{5}\right),\left(v_{2}, v_{4}\right)\left(v_{0}, v_{7}\right),\left(v_{2}, v_{6}\right)\left(v_{0}, v_{3}\right)$ and $\left(v_{4}, v_{6}\right)\left(v_{0}, v_{3}\right)$ are all automorphisms of $S_{15}$. So, we may assume that $\left(a_{1}, a_{2}\right)=$ $\left(v_{1}, v_{5}\right),\left(b_{1}, b_{2}\right)=\left(v_{2}, v_{0}\right),\left(c_{1}, c_{2}\right)=\left(v_{4}, v_{7}\right)$ and $\left(d_{1}, d_{2}\right)=\left(v_{6}, v_{3}\right)$. Then (up to an equivalence), $\varphi=f$. The theorem now follows from Example 2.1.

Remark 6.3. Some of the steps in the proofs of the lemmas in $\S 4$ and 5 are similar to the others. Hence we have omitted these details for the sake of brevity. Complete proofs are available with the authors.

\section{References}

[1] Altshuler A, Combinatorial 3-manifolds with few vertices, J. Comb. Th. (A) 16 (1974) $165-173$

[2] Altshuler A and Steinberg L, An enumeration of combinatorial 3-manifolds with nine vertices, Disc. Math. 16 (1976) 91-108

[3] Bagchi B and Datta B, A structure theorem for pseudomanifolds, Disc. Math. 168 (1998) $41-60$

[4] Cervone D P, Vertex-minimal simplicial immersions of the Klein bottle in three space, Geo. Dedicata 50 (1994) 117-141

[5] Datta B, Two dimensional weak pseudomanifolds on seven vertices, Bol. Soc. Mat. Mexicana 5 (1999) 419-426

[6] Datta B and Nilakantan N, Equivelar polyhedra with few vertices, Discrete Comput Geom. 26 (2001) 429-461

[7] Kühnel W, Triangulations of manifolds with few vertices, in Advances in differential geometry and topology (ed) F Tricerri (Singapore: World Scientific) (1990) pp 59-114

[8] Munkres J R, Elements of algebraic topology (California: Addison-Wesley) (1984)

[9] Ringel G, Wie man die geschlossenen nichtorientierbaren Flächen in möglichst wenig Dreiecke zerlegen kann, Math. Ann. 130 (1955) 317-326

[10] Rourke C P and Sanderson B J, Introduction to piecewise-linear topology (Berlin: Springer-Verlag) (1982)

[11] Spanier E H, Algebraic Topology (Berlin: Springer-Verlag) (1982)

[12] Thomas R, An up date on the four-color theorem, Notices Amer. Math. Soc. 45 (1998) 848-859 